\title{
Space weather impacts on satellites and forecasting the Earth's electron radiation belts with SPACECAST
}

\author{
R. B. Horne, ${ }^{1}$ S. A. Glauert, ${ }^{1}$ N. P. Meredith, ${ }^{1}$ D. Boscher, ${ }^{2}$ V. Maget, ${ }^{2}$ \\ D. Heynderickx, ${ }^{3}$ and D. Pitchford ${ }^{4}$
}

Received 8 October 2012; revised 15 December 2012; accepted 2 January 2013; published 10 April 2013.

[1] Satellites can be damaged by high energy charged particles in the Earth's radiation belts and during solar energetic particle (SEP) events. Here we review the growing reliance on satellite services, new vulnerabilities to space weather, and previous events that have led to loss of service. We describe a new European system to forecast the radiation belts up to $3 \mathrm{~h}$ ahead, which has three unique features: first, it uses physics-based models, which include wave-particle interactions; second, it provides a forecast for the whole outer radiation belt including geostationary, medium, and slot region orbits; third, it is a truly international effort including Europe, United States, and Japan. During the 8-9 March 2012 storm and SEP event, the models were able to forecast the $>800 \mathrm{keV}$ electron flux to within a factor of 2 initially, and later to within a factor of 10 of the GOES data. Although ACE and GOES data became unreliable during the SEP event, the system continued forecasting without interruption using ground-based

magnetometers. A forecast of the $24 \mathrm{~h}$ electron fluence $>2 \mathrm{MeV}$ is used to provide a risk index for satellite operators. We show that including wave-particle interactions for $L^{*}>6.5$ improves the agreement with GOES data substantially and that a fast inward motion of the magnetopause to $L^{*}<8$ is related to rapid loss of relativistic electrons at geostationary orbit. Thus, we suggest that better wave-particle models and better coupling between the solar wind and the models of the magnetopause and radiation belts should lead to better forecasting.

Citation: Horne, R. B., S. A. Glauert, N. P. Meredith, D. Boscher, V. Maget, D. Heynderickx, and D. Pitchford (2013), Space weather impacts on satellites and forecasting the Earth's electron radiation belts with SPACECAST, Space Weather, 11, 169-186, doi:10.1002/swe.20023.

\section{Introduction}

[2] As of May 2012, there are 994 operational satellites on orbit of which 419 are in geosynchronous orbit (GEO), 69 in medium earth orbit (MEO), 35 in elliptical orbit, and 471 in low earth orbit (LEO) [SIA, 2012]. This represents a large growth in the satellite industry since 2002 when the number of satellites at GEO was approximately 200 [The Futron Corporation, 2002] (for an illustration of satellite orbits in

\footnotetext{
${ }^{1}$ British Antarctic Survey, Madingley Road, Cambridge CB3 0ET, UK.

${ }^{2}$ The French Aerospace Laboratory, F-31055, Toulouse, France.

${ }^{3}$ DH Consultancy BVBA, Leuven, Belgium.

${ }^{4}$ Klostergartenstrasse 67, Leiwen 54340, Germany.

Corresponding author: R. B. Horne, British Antarctic Survey, Natural Environment Research Council, Madingley Road, Cambridge, CB3 0ET, England, UK. (r.horne@bas.ac.uk)
}

(02013. American Geophysical Union. All Rights Reserved. 1542-7390/13/10.1002/swe.20023 relation to the Earth, see Figure 1). Today satellites are usedfor many different applications, including communications, global navigation, positioning, meteorology, Earth observation, science, technology, security, and defense. In 2011, the total revenue from the space industry as a whole was US \$289.8 billion, and the largest sector was satellite telecommunications at US $\$ 177.3$ billion. Approximately 381 satellites $(38 \%)$ are used for commercial communications and the largest consumer revenue is for satellite TV (US $\$ 88.6$ billion) followed by satellite radio (US $\$ 3.0$ billion) and broadband (US $\$ 1.2$ billion) [SIA, 2012]. These industry statistics show the importance of the space industry to modern economies and our reliance on satellite services. That reliance is also growing in unexpected ways and is producing vulnerable dependencies, for example, automated high frequency trading now accounts for more than $60 \%$ of equity trading on the London and New York stock markets and uses GPS signals to timestamp transactions with millisecond to microsecond time resolution [The Economist, $25 \mathrm{Feb}, 2012]$. 


\section{The Earth's Electron Radiation Belts}

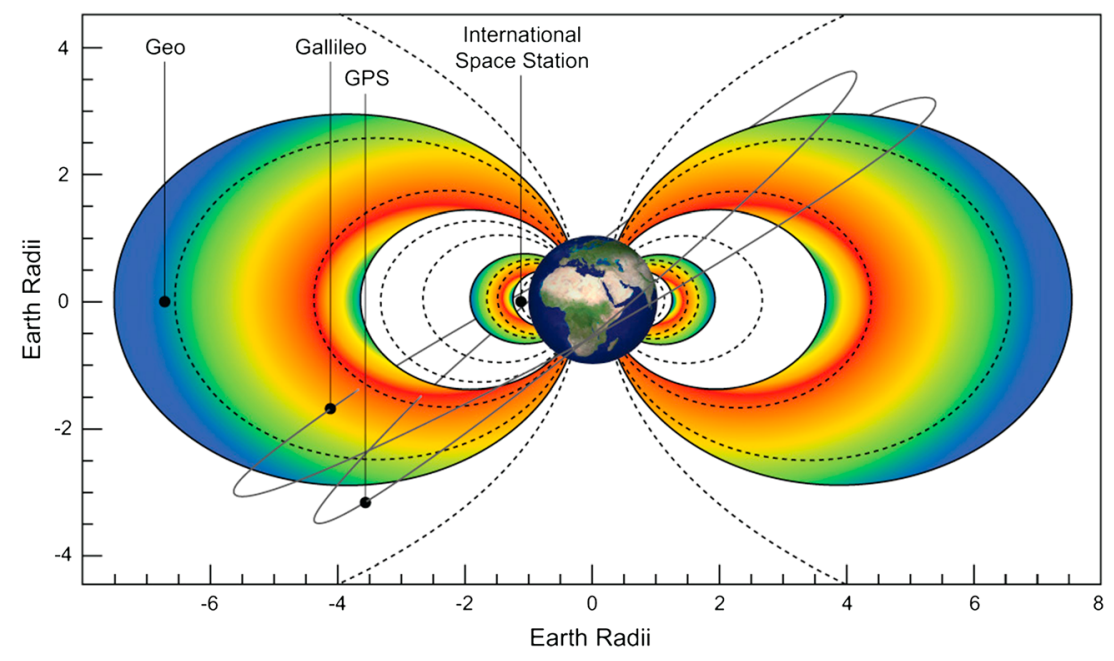

Figure 1. Different types of satellite orbits in relation to the Earth and the Earth's radiation belts. Most telecommunications satellites are in GEO, GNSS satellites such as Galileo and GPS are in MEO, and the international space station is in low Earth orbit (LEO). The slot region lies between the inner and outer radiation belts.

[3] Over the last 10 years, there have been several developments that have increased the vulnerability of satellites to space weather, particularly high energy charged particles. Radiation-hardened components have become increasingly difficult to obtain, and so there has been increased use of commercial off the shelf components in satellite design (http://www.sstl.co.uk/About-SSTL/SSTL-Approach), which may be more susceptible to radiation damage. Similarly, electronic components have become more susceptible to radiation damage as a result of miniaturization, and higher solar array operating power may increase the risk of damage from spacecraft-plasma interactions. These developments have been offset by better design, duplication of circuits with error correction techniques, and more sophisticated engineering models, but these advances have yet to be tested in a major geomagnetic storm or solar energetic particle (SEP) event which can last for several days.

[4] Over the last few years, there has been an extended period of solar minimum, which has led to a relatively benign space environment, but solar activity is now increasing and the number of sunspots is expected to reach a maximum some time in 2013. More importantly, the number of geomagnetic storms triggered by solar activity tends to peak some 2 years after the peak in the sunspot cycle and, based on results from the last solar cycle, will generally pose a much harsher and dynamic radiation environment than we have experienced over the last 5 or 6 years. More than 110 satellites have been launched since 2004 with new design and new technology, and thus there are a significant number of satellites that have never encountered conditions associated with solar maximum. The next several years or so following solar maximum will provide an important test of the new design and mitigation measures.
[5] More generally, the prospect of another severe space weather event, as big as the 1859 Carrington event [e.g., Tsurutani et al., 2003], has raised concern at government level. Like volcanoes and tsunamis, severe space weather is a low probability but high impact event. The loss of revenue from satellite services alone, from an event three times bigger than the Carrington event, has been estimated to be as high as US\$30 billion [Odenwald and Green, 2007], and this is without considering the impact on the financial and other industries that rely on satellite services or other societal effects [NAS, 2008; Cannon et al., 2013]. Severe space weather, corresponding to a 1 in a 100 year event, is now on the UK National Risk Register of Civil Emergencies [Cabinet Office, 2012] and warrants further study of all areas of space weather.

\subsection{Space Weather Effects on Satellites}

[6] Space weather can affect satellites in several different ways. Solar EUV and Joule heating during geomagnetic storms heat the atmosphere and increase drag on satellites in low Earth orbit and can cause uncontrolled reentry. However, at higher orbits, the most important space weather danger is from high energy charged particles [Hastings and Garrett, 1996]. Cosmic rays and SEPs can penetrate electronic components causing single event effects [e.g., Koons and Fennell, 2006]. They cause ionization in insulating layers, which can lead to leakage currents; increase noise and additional power consumption, which can corrupt memory circuits and in some cases, unless action is taken, cause burnout and component failure. In many cases, fault-tolerant software and duplicate circuits can correct these errors, but a major SEP event can cause a challenging environment with many malfunctions in a short space of time. 
[7] SEP events also cause displacement damage in electronic components, whereby atoms are knocked out of the crystal lattice leading to defects, charge trapping, and reduced gain in transistors. This is particularly important for solar arrays and leads to a degradation of solar array power over the lifetime of the satellite. For example, solar array power may degrade by $1 \%-2 \%$ per year depending on the type of solar cells [Brekke, 2004], whereas one large SEP event can cause $2 \%$ power loss [Odenwald and Green, 2007] in modern GaAs/Ge solar arrays. The total ionizing dose from all space radiation, electrons, and ions also affects the efficiency of surface materials such as solar array coatings, thermal control, and electronic components especially charged coupled devices. Total ionizing dose affects components from the surface to deep inside the spacecraft, and thus particles with a broad energy range from a few electron volts to several million electron volts (MEV) are a concern.

[8] High energy $(\mathrm{MeV})$ electrons in the Earth's radiation belts pose an important risk of internal satellite charging [Frederickson, 1996; Wrenn, 1995; Gubby and Evans, 2002]. Radiation belt electrons can penetrate the outer skin of the spacecraft and accumulate in insulating materials such as cables and ungrounded conductors and cause internal electrostatic charging. Internal electrostatic charging can give rise to very high electric fields which, if they exceed the break-down field of the dielectric, can cause an electrostatic discharge (ESD), resulting in permanent damage to the dielectric, component failure, phantom commands causing uncontrolled behavior of the spacecraft [Wrenn et al., 2002], and other undesirable effects. By 1996, more than 20 satellites had been damaged by internal ESD [Wrenn and Smith, 1996], and more recent research shows that there is a statistically significant correlation between satellite anomalies and elevated daily electron fluence $>2$ $\mathrm{MeV}$ at geostationary orbit [ucci et al., 2005]. It is also important to note that even if an ESD is not triggered during a major storm the time constant for the charge to decay may be years, depending on the dielectric material [Bodeau, 2010; Fennell et al., 2010], and thus the spacecraft may be susceptible to a subsequent but smaller space weather event. Radiation-hardened components and shielding sensitive components with aluminum are often used to protect against internal charging, but this can be expensive because of the added mass and increased launch costs and thus there is always a cost balance tradeoff.

[9] Surface charging is another important risk for satellites [Hastings and Garrett, 1996; Lanzerotti et al., 1998a; Koons and Fennell, 2006]. In sunlight, the satellite potential may float positive due to a net balance of currents between the satellite and the plasma due to the primary incident electrons and ions, backscattered and secondary emitted particles, and photoelectron emission. A sheath forms around the spacecraft which can be distorted by electric fields, satellite motion through the plasma and thruster firings. Substorms inject large clouds of medium energy $(\sim 10-20 \mathrm{keV})$ electrons, which are much more energetic than the ambient thermal electrons at geostationary orbit. The substorm-injected electrons disturb the current balance and charge the satellite surfaces to a large negative potential. As only half the spacecraft can be in sunlight and different materials have different photoelectron emission properties, components can charge to different potentials, as high as $10 \mathrm{kV}$. Again, if the electric field exceeds the breakdown field of the surface material, either along the surface or through the material to the spacecraft frame, an ESD can occur and cause damage to surface materials and the underlying components. Making the satellite surfaces electrically conducting is one possible mitigation, but this is not always possible. Typically, most satellite anomalies due to surface charging at geostationary orbit occur at night and early dawn [Fennell et al., 2001; Gubby and Evans, 2002; O'Brien, 2009], which coincides with the most probable region for substorm injection and electron drift toward dawn under the influence of magnetospheric electric fields.

\subsection{Satellite Anomalies and Loss of Service}

[10] The number of satellites that have been affected by space weather is very difficult to assess. However, there are several well-known examples that illustrate the importance of space weather. For example, on 20 January 1994, Intelsat $\mathrm{K}$, Anik E1, and Anik E2 suffered anomalies resulting in the loss of attitude control. Intelsat K and Anik E1 were recovered after a few hours, but Anik E2 was only recovered after 6 months. One hundred thousand customers had to repoint their satellite dishes. The anomalies occurred during a period of enhanced relativistic $(>2 \mathrm{MeV})$ electron flux [Baker, 2001], and the most likely cause was identified as an ESD due to internal charging by radiation belt electrons.

[11] On 11 January 1997, Telstar 401 suffered an abrupt telemetry and communications failure resulting in a total loss. The anomaly was traced to an ESD that occurred during a space weather event associated with the passage of a magnetic cloud past the Earth's magnetosphere [Lanzerotti et al., 1998b].

[12] On 19 May 1998, Galaxy IV suffered an anomaly that caused the satellite to rotate and lose fixed orientation, and the backup system also failed. The satellite was unable to maintain a stable Earth communications link and was lost from useful service, affecting 30 million customers or more relying on telephones, pagers, and other communications devices [Baker et al., 1998]. The anomaly occurred during the highest electron enhancement in 1997-1998, which suggested that internal charging was the likely cause of an ESD [Baker, 2001]. However, it was shown years later that after similar component failures on other satellites of the same type that the loss of Galaxy IV was unlikely to be due to space weather [Bodeau, 2007]. The key issue here was and is the need for environmental data.

[13] Between 23 October and 6 November 2003, 47 satellites reported malfunctions, and the Midori 2 (also known as ADEOS) scientific satellite costing US $\$ 640 \mathrm{~m}$ was a total loss [Webb and Allen, 2004]. More than 10 satellites were out of action for more than 1 day. These anomalies occurred during the so-called Halloween storm, where there were very large changes in the radiation belts and where an SEP event took place at the same time. 
[14] On 5 April 2010, Galaxy 15 suffered an anomaly and would not respond to ground control [Allen, 2012]. The satellite drifted around geostationary orbit, and several other satellites had to take evasive action to avoid transmission interference. In this case, there was also a risk of collision at GEO. The satellite ran out of power and was brought back under control in January 2011. Although solar activity was low, the anomaly occurred during a large magnetic storm at the Earth [Ferguson et al., 2011; Denig et al., 2011].

[15] There are also examples of more recent events. For example, on 7 March 2012, the Sky Terra 1 satellite reported problems resulting in loss of service for a few days [http://www.flightglobal.com/blogs/hyperbola/ 2012/03/spaceway-3-temporarily-knocked.html]. In this case, there was only a moderate geomagnetic storm, but magnetometers at Halley research station in the Antarctic and satellites in geostationary orbit indicated multiple substorms and plasma injections, which are known to cause surface charging. Spaceway 3 and GOES 15 also reported outages in March 2012 for a few hours and a few days, respectively, indicating that anomalies are still an important issue.

[16] It is very difficult to determine whether space weather is responsible for a satellite anomaly. In some cases, this may be due to the lack of availability of housekeeping telemetry as a result of the anomaly, the lack of adequate information on the space environment, or the difficulty of assessing cause and effect remotely. There is also the question as to why one spacecraft might suffer an anomaly while many others of the same basic design continue operating successfully [Lanzerotti et al., 1998b; Bodeau, 2007]. This could be due to differences in the final specification put forward by the purchaser as well as the fact the risk of space weather damage varies considerably with magnetic local time [e.g., O'Brien, 2009] as well as by orbit type. By the same token, it is very difficult to rule out space weather as the cause, particularly for orbits where there are no in situ measurements, or if measurements are only available at magnetic local times many hours different to that of the problem spacecraft.

[17] Data on satellite anomalies is commercially sensitive due to the large sums of money involved in the construction and operation of satellites in a very competitive industry. For example, a modern telecommunications satellite may cost around US\$250 million to build and carry up to 100 transponders. Satellite operators lease the transponders for typically US $\$ 2$ million per transponder per year for a design life of 15 years. As a result, data on satellite anomalies are confidential and very difficult to obtain for scientific analysis for the fear that a competitor may take advantage. This makes it very difficult to make the case for basic research that will ultimately support the industry. What is clear from the above analysis is that satellite anomalies still result in loss of services and in some cases total satellite loss and thus remain an important issue.

\subsection{Benefits of a Forecasting System}

[18] While better design can help protect satellites, test facilities cannot fully replicate the space environment, and unexpected environmental sensitivities still occur that can cause anomalies. Therefore, some measure of forecasting and warning of the space radiation environment is highly desirable. A reliable forecasting system has several benefits. First, for satellite operators and satellite design and construction companies, a forecasting system that includes measurements and dynamic modeling should help identify the cause of an anomaly rapidly via the reconstruction the space environment during the event, particularly for locations where there are no measurements. Second, for satellite operators, it provides situation awareness that can be used to raise the alertness level of operators on the ground to deal with any potential problems, and given enough warning, to have more staff available. Third, it enables satellite operators to take action to mitigate the risk of service interruptions, for example, by switching off nonessential systems, by rescheduling orbit maneuvers and software upgrades, and by ensuring spare capacity is immediately available to reroute communications traffic. Space insurance companies, who insure approximately 176 satellites at GEO [Kunstadter, 2012], may also benefit as satellite operators are required to take all reasonable precautions to protect their assets.

[19] The purpose of this article is to introduce a new system to forecast the Earth's dynamic radiation belts, which has been developed as part of the SPACECAST project under the European Union Framework 7 program [www.fp7-spacecast.eu]. The system utilizes scientific and operational data from ground and space, state-of-the-art research models, and a distributed system of information and modeling centers that provides robustness. The system is automatic, is updated every hour, and runs $24 \mathrm{~h}$ a day, 7 days a week, 365 days a year. The system has three unique features. First, it uses physics-based models, which include wave-particle interactions to provide the forecasts. Second, it provides a forecast for the whole of the outer electron radiation belt, including geostationary orbit where most commercial satellites operate; MEO where the global navigation satellite systems (GNSS) operate such as GPS (USA), Galileo (Europe), GLONASS (Russia), and Compass (China); and the slot region between the inner and the outer radiation belts, where the radiation environment is usually benign but can increase rapidly during storms and where the number of satellites is expected to grow (Figure 1). Third, it is a truly international effort, using data from U.S. satellites, geomagnetic indices from Europe and Japan, a network of European radiation belt forecasting models, and a database system developed by the European Space Agency.

\section{SPACECAST Forecasting System}

[20] The concept behind the SPACECAST forecasting system is that data collected by the ACE spacecraft at the L1 position (some $1.5 \times 10^{6} \mathrm{~km}$ from the Earth toward the 
Sun) could be used to drive forecasting models. For a typical solar wind velocity of $400 \mathrm{~km} \mathrm{~s}^{-1}$, it takes about $60 \mathrm{~min}$ for the solar wind to travel from L1 to the Earth's magnetopause. However, the travel time can be much shorter during fast solar wind streams ( $30 \mathrm{~min})$ and shorter still for coronal mass ejections (CMEs) as the fastest CME speed can be in excess of $2000 \mathrm{~km} \mathrm{~s}^{-1}$ [Cliver et al., 1990]. Because the solar wind drives the magnetosphere and the magnetosphere drives the radiation belts, data from the L1 position provides some advanced information to predict how the radiation belts will respond. Actually, the timescale to forecast ahead reliably is more complicated than this simple calculation, as discussed below.

[21] There is one key factor in using the ACE data for forecasting as opposed to remote observations of CMEs, which might give a longer lead time. ACE measures the polarity of the interplanetary magnetic field and thus the efficiency of energy coupling into the geomagnetic field via magnetic reconnection, particle transport via convection and inductive electric fields inside the magnetosphere, and the likelihood of substorms and full scale geomagnetic storms. These processes affect the source, transport, acceleration, and loss of radiation belt electrons. Thus, the ACE data increase the reliability of the radiation belt forecasts and reduces the possibility of false alarms.

[22] Figure 2 shows a schematic view of the data collection and flow within the forecasting system. Here, we concentrate on the high energy electron forecasts only; nowcasts for the low energy $(10 \mathrm{keV})$ electron and SEP events will be described in another publication. Data from six different sites are accessed and downloaded to a central server hosted in Belgium. In all these data, flow operations data are requested and downloaded at times when it is required; it is not sent at a fixed time. This enables a more robust system. The Swedish Institute of Space Physics, Lund, uses ACE data to forecast the $K_{\mathrm{p}}$ index (http://rwc.lund.irf.se/ $\mathrm{rwc} / \mathrm{kp} /$ index.php). This forecast of $K_{\mathrm{p}}$ is used by SPACECAST to drive the forecasting models. Forecasts of other indices, such as AE and the polar cap index, are being investigated and may provide a better driver for the models, but these have not yet been implemented. Occasionally, the $K_{\mathrm{p}}$ forecast is not available in which case an estimated $K_{\mathrm{p}}$ index is obtained from the British Geological Survey (BGS) and used to drive the models. This provides some measure of resilience to the system. Other supporting data, which are not used to make the forecasts, are also accessed and displayed on our Web site, including the quick look $D_{\text {st }}$ index from the Regional Warning Centre, Kyoto, Japan; the solar wind velocity and magnetic field from the ACE SWEPAM and MAG instruments; the high energy electron data from the GOES and POES satellites from the National Oceanic and Atmosphere Administration; and the magnetometer data from the Halley Research Station in Antarctica. These supporting data, such as the electron integral flux at GEO from the primary GOES satellite (currently GOES 13), provide a valuable cross check on the forecasts

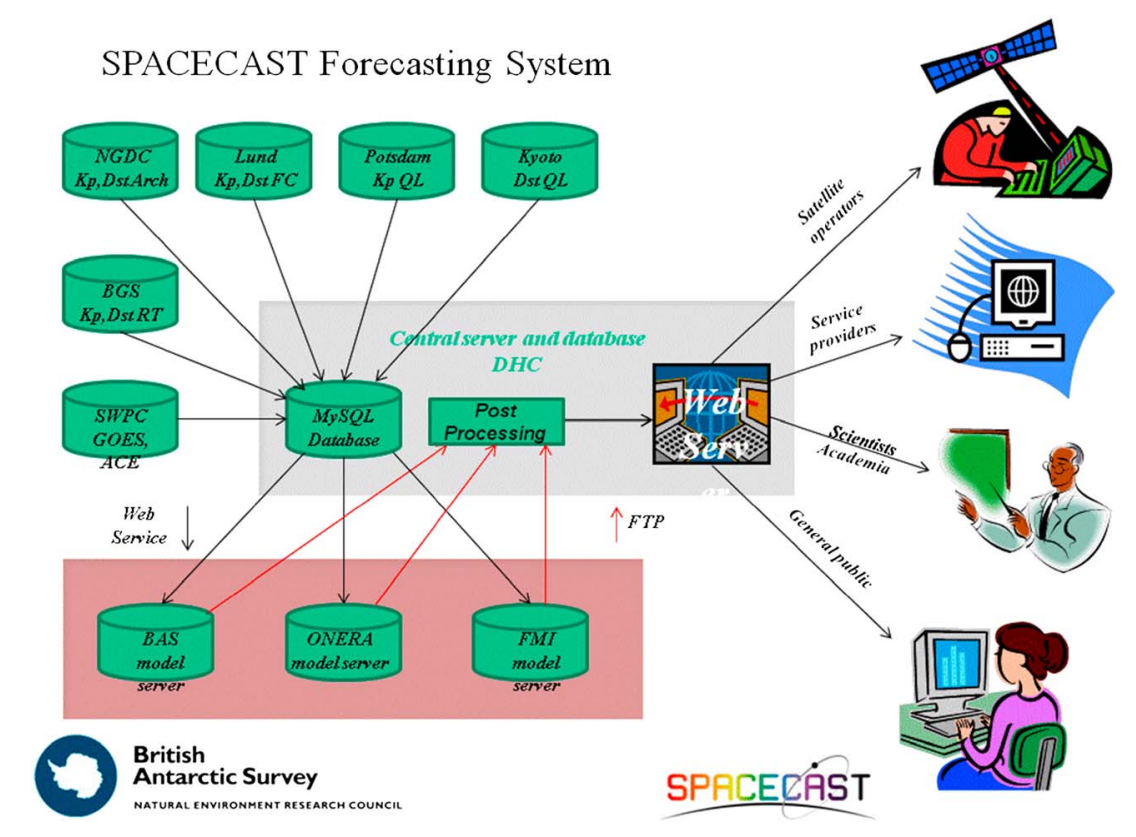

Figure 2. Illustration of the SPACECAST forecasting system. Data are collected from several different satellite and ground-based data centers, merged and processed into a central database. The processed data are then collected by different modeling centers and used to run two independent models to forecast the high energy electron radiation belt flux. Forecasting results are collected by the central server and processed to provide the Web displays for several types of users. The process works automatically. 
and by bringing them together into one display helps interpretation. The $K_{\mathrm{p}}$ index is also accessed from an archive at the Helmholz Centre, Potsdam, Germany, and $D_{\text {st }}$ index from the archive at the National Geophysical Data Centre, Boulder, USA.

[23] The real-time data are accessed and processed every hour and made available for the models. For the high energy radiation belts, the data are accessed by modeling centers at the British Antarctic Survey, Cambridge, UK, and the French Aerospace Laboratory (ONERA), Toulouse, France. A modeling center at the Finish Meteorological Institute, Helsinki, Finland, is concentrating on the lowerenergy electrons, which will be described elsewhere.
Because $K_{\mathrm{p}}$ is a $3 \mathrm{~h}$ index, the forecast of $K_{\mathrm{p}}$ enables the models to provide a forecast of the radiation belts up to 3 $\mathrm{h}$ ahead. However, as the forecast of $K_{\mathrm{p}}$ is updated every hour, the time resolution is much better than $3 \mathrm{~h}$. In effect, we provide a $3 \mathrm{~h}$ forecast updated every hour.

[24] The results of the model runs are accessed centrally by the data server. The results are combined with the supporting data and presented on the Web page as multipanel plots (see Figure 3) and movies, one for each model. These plots are available for the general public (www.fp7spacecast.eu). SPACECAST also calculates a risk index to indicate the risk of internal satellite charging for satellites at geostationary orbit, $\mathrm{MEO}$ representative of that used

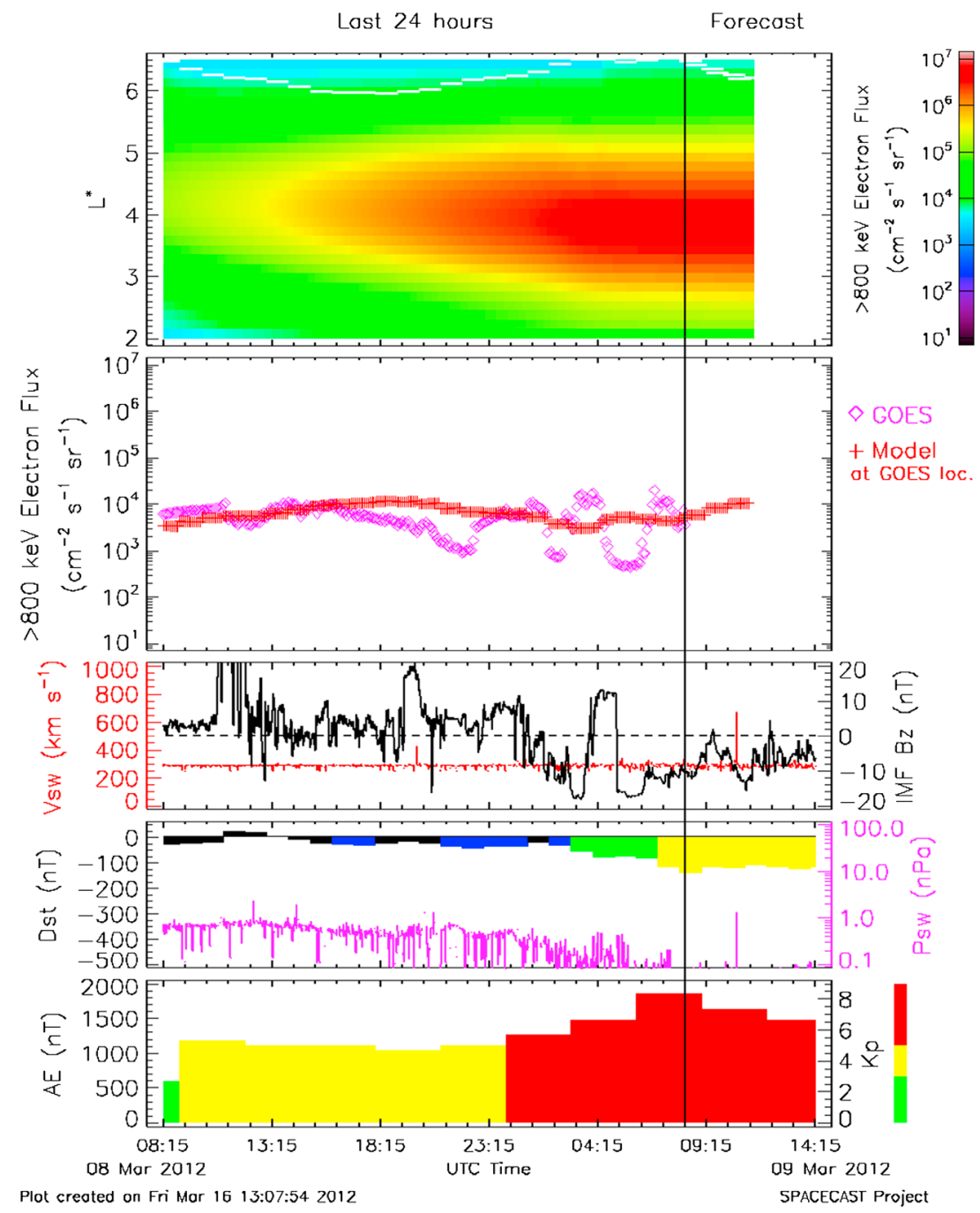

Figure 3. Forecasting results for the 8 and 9 March 2012. From top to bottom, (a) the $>800 \mathrm{keV}$ electron flux color coded as a function of $L^{*}$ and time. The white line shows the location of the GOES 13 satellite, (b) the model results at the location of GOES (red crosses), and the GOES measurements (pink diamonds), (c) the $z$ component of the interplanetary magnetic field (IMF $\mathrm{Bz}$ ) and solar wind velocity from $\mathrm{ACE},(\mathrm{d})$ the provisional $D_{\mathrm{st}}$ index and the solar wind speed from ACE, and (e) the $K_{\mathrm{p}}$ index. The $3 \mathrm{~h}$ forecast is to the right of the vertical line in Figures $3 \mathrm{a}$ and $3 \mathrm{~b}$. An additional $3 \mathrm{~h}$ data have been added to the bottom panels. 
by the GNSS satellites and the slot region. The risk index is based on a calculation of the $24 \mathrm{~h}$ electron fluence $>2$ $\mathrm{MeV}$. More details are given in the following section.

\section{Radiation Belt Models}

[25] At the heart of the forecasting system are the two radiation belt forecasting models. These forecasting models have been adapted from research models: the British Antarctic Survey (BAS) radiation belt model and the Salammbô model at ONERA [Beutier and Boscher, 1995; Varotsou et al., 2005, 2008]. Both models are based on a diffusion equation (given in Appendix A), but they are completely independent. Transforming these research models into forecasting models raises new challenges. For example, in research studies, the boundary conditions can usually be obtained from satellite data after the event and one can simulate the whole radiation belts to examine the physics. However, there are no observations to set the boundary conditions for forecasting, and indeed the object is to forecast the electron flux ahead of time. In the SPACECAST forecasting system, we use a forecast of the $K_{\mathrm{p}}$ index to drive the models. The diffusion coefficients used in the models are scaled by the $K_{\mathrm{p}}$ index and the phase space density $f$ at the outer boundaries varies according to the value of $K_{\mathrm{p}}$, as described in the following paragraphs. This defines the whole simulation domain.

[26] In the SPACECAST system, only the outer electron radiation belt and the slot region are included in the models. Although the inner belt is very important for satellites in LEO, there are relatively few observations compared with the outer belt, and the outer belt is much more dynamic. The inner belt will be addressed at a later date.

[27] In outline, the models solve a diffusion equation to find the evolution of the electron phase space density $f$ $\left(\alpha, E, L^{*}, t\right)$ as a function of pitch angle $\alpha$, energy $E, L^{*}$, and time. The phase space density is then converted into the differential $J(E, \alpha)$ and integral $J_{I}\left(E>E_{0}, \alpha\right)$ electron flux for comparison with satellite data. The models include several physical processes, including electron pitch angle and energy diffusion due to wave-particle interactions, radial transport across the magnetic field due to ultra low frequency (ULF) waves, and Coulomb collisions with atmospheric gases. Wave-particle interactions can both accelerate electrons to relativistic energies, which increases the radiation belts [Horne and Thorne, 1998; Summers et al., 1998; Horne et al., 2005a, 2005b; Horne, 2007] and cause losses into the atmosphere which depletes the radiation belts [Lyons and Thorne, 1973; Meredith et al., 2009], depending on the solar wind driver. A model for whistler mode chorus waves, which are very important for electron acceleration, is used here where the waves cover the region outside the plasmapause out to $L^{*}=6.5$ and extend along the magnetic field lines from the equator up to $30^{\circ}$ in latitude [Meredith et al., 2003]. The $30^{\circ}$ chorus model has been used in a previous global modeling study [Albert et al., 2009] and a $15^{\circ}$ chorus models in other studies [Varotsou et al., 2005, 2008; Fok et al., 2008]. Inside the plasmasphere, the BAS model uses a model for plasmaspheric hiss, whereas the Salammbô model uses the model based on Abel and Thorne [1998]. The radial diffusion model is the same as that used by Brautigam and Albert [2000], except that the electrostatic radial diffusion coefficient for $90^{\circ}$ pitch angle is applied to all pitch angles.

[28] The models use a coordinate system that is based on the three adiabatic invariants associated with electron cyclotron motion around the magnetic field, bounce motion along the magnetic field between mirror points in the northern and southern hemisphere, and drift motion around the Earth. This enables the models to take advantage of the conservation laws associated with the adiabatic invariants and thus reduce computation. In practice, the equations are solved on two computer grids, one for radial diffusion at constant first and second invariants and the other on an orthogonal grid for equatorial pitch angle and energy diffusion at constant $L^{*}$ for a dipole field. This ensures the appropriate conservation [e.g. Shprits et al., 2009]. The computations are transformed between the two grids every half time step. The simulation domain is a rectangular box where for the BAS model, the $L^{*}$ range is from 2 to 6.5 (1-8 for Salammbô) and the equatorial pitch angle range is from $0^{\circ}$ to $90^{\circ}\left(2^{\circ}-90^{\circ}\right.$ for Salammbô). For the BAS model, the first invariant $\mu$ range is from 10 to $36,000 \mathrm{MeV} / \mathrm{Gauss}$, which corresponds to electron energies between $11 \mathrm{keV}$ and $6 \mathrm{MeV}$ at $L^{*}=6.5$, and energies between $300 \mathrm{keV}$ and $37 \mathrm{MeV}$ at $L^{*}=2$. For the Salammbô model, $\mu$ is from 0.16 to $48,000 \mathrm{MeV} / \mathrm{Gauss}$, corresponding to energies between $98 \mathrm{eV}$ and $5.0 \mathrm{MeV}$ at $L^{*}=8$ and energies between $48 \mathrm{keV}$ and $123 \mathrm{MeV}$ at $L^{*}=1$.

[29] In the BAS model, the boundary conditions are set so that the gradient in the equatorial pitch angle distribution is zero at $0^{\circ}$ and $90^{\circ}$, and $f=0$ at the high energy boundary for all $L^{*}$ and $\alpha$. At the low energy boundary and at the inner and outer $L^{*}$ boundaries, $f$ varies with $K_{\mathrm{p}}$, where the value of $f$ at each $L^{*}$ was determined from the average electron phase space density measured by the CRRES satellite over the whole mission lifetime for different levels of $K_{\mathrm{p}}$. For the Salammbô model $f=0$ at $\alpha=2^{\circ}$, the gradient in equatorial pitch angle is zero at $90^{\circ}$, and $f=0$ at the inner $L^{*}$ boundary for all $\alpha$ is constant along the lower $\mu$ boundary for all $L^{*}$ and varies with $K_{\mathrm{p}}$ at the outer $L^{*}$ boundary.

[30] The electron data used to set the boundary conditions were first transformed into dipole coordinates $\left(L^{*}\right)$ using the International Radiation Belt Environment Modeling library software (www.irbem.sf.net) using the IGRF field and the Olsen-Pfitzer quiet time field model. The Olsen-Pfitzer model was chosen as it has been shown to be a good average external magnetic field model when compared with measurements [Friedel et al., 2005] and has recently been adopted by the Panel for Radiation Belt Environment Modelling for improving space radiation models. This transformation takes into account the nondipole features of the geomagnetic field. The transformation works best on the dayside of the Earth but can still lead to large uncertainties on the nightside [e.g., Green and Kivelson, 2004] and during large geomagnetic storms. 
The GOES satellite data at GEO are not used in the forecasting but are used to verify the model results and therefore are also transformed into $L^{*}$.

[31] Both research models have been tested against radiation belt variations measured by the CRRES satellite. They show that radial diffusion alone cannot account for electron flux variations observed in the outer radiation belt and that whistler mode chorus waves are very effective in providing electron acceleration in the heart of the outer radiation belt [Varotsou et al., 2005, 2008]. When radial diffusion is combined with chorus wave acceleration, there is much better agreement between the models and the observations. Similar results have also been found by various modeling studies [Shprits et al., 2006a, 2006b; Fok et al., 2008; Albert et al., 2009; Varotsou et al., 2005, 2008; Su et al., 2010].

\section{The Storm of 8-9 March 2012}

[32] Figure 3 shows an example of modeling the high energy electron radiation belts for the period 08:15 on the 8 March 2012 to 14:15 UT on the 9 March 2012. The top panel shows the $>800 \mathrm{keV}$ integrated electron flux obtained from the models, color coded, as a function of time and $L^{*}$. The models were initialized at the beginning at $08: 15$ by taking the average value of $K_{\mathrm{p}}$ for the 3 days before the start of the simulation and run to a steady state solution. The models were then run for $27 \mathrm{~h}$ according to the time series of $K_{\mathrm{p}}$. The first $24 \mathrm{~h}$ used the measured values of $K_{\mathrm{p}}$, while the final $3 \mathrm{~h}$ used the forecast value of $K_{\mathrm{p}}$. Thus, the forecast (08:15-11:15 UTC) is shown to the right of the vertical line. The white line in the top panel shows the location of the GOES 13 satellite in $L^{*}$ coordinates. Although GOES 13 is at geostationary orbit, as the satellite moves in MLT, the magnetic field strength changes due to the nondipole components of the Earth's magnetic field, with higher magnetic field strength usually on the dayside of the Earth. Once the orbit is transformed into $L^{*}$, the orbit appears as a curved arc where the region of lowest $L^{*}$ corresponds to GOES being on the dayside. Under quiet conditions, the electron flux at GEO is usually higher on the dayside as the outer radiation belt extends to larger radial distance due to conservation of the third adiabatic invariant.

[33] During the simulation, the electron flux near $L^{*}=4$ increased by more than an order of magnitude due to a combination of chorus wave electron acceleration and inward radial diffusion. Note that the simulation did not show a large increase in electron flux near geostationary orbit. This may be partly due to our choice of outer boundary conditions, although the flux at the outer boundary increases with $K_{\mathrm{p}}$ in our model, and partly due to the nature of the acceleration process. The simulation showed that the GNSS-type orbits were more at risk from the increase in electron flux than GEO. The $K_{\mathrm{p}}$ and the $D_{\text {st }}$ indices (fifth panel), which are often used to measure the strength of the geomagnetic storm, reached $K_{\mathrm{p}}>8$ on 9 March 2012 and $D_{\text {st }} \approx-133 \mathrm{nT}$ (fourth panel), both indicating a strong geomagnetic storm [Loewe and Prolss, 1997].
Before this, on 7 March 2012, there was a moderate storm with $-50>D_{\text {st }}>-100 \mathrm{nT}$.

[34] The second panel shows the integral electron flux measured by GOES 13 compared with that obtained from the model. The $3 \mathrm{~h}$ forecast is given to the right of the black vertical line at $08: 15$. The model is able to reproduce the data to within a factor of 2 for a period of about $10 \mathrm{~h}$ from the beginning of the event. After about 23:15, there are large variations in the measured electron flux, which are not captured in the model, but the model is able to reproduce the data to within a factor of 10 .

[35] The third panel shows the data obtained from the ACE spacecraft. The IMF Bz had large negative excursions, particularly after 23:15, indicating energy transfer into the magnetosphere. This is also reflected in the gradual decrease in the $D_{\text {st }}$ index, which indicates plasma injection toward the Earth and buildup of the ring current, and the increase in $K_{\mathrm{p}}$, which indicates enhanced disturbances in the geomagnetic field. The solar wind velocity is almost constant, which appears unphysical, and suggests that the satellite instrument could not measure the bulk velocity accurately at this time. Thus, we have made no attempt to shift the solar wind data by the delay time between the L1 and the magnetopause. The problem with the solar wind velocity also suggests that the dynamic solar wind pressure, calculated from $\rho v^{2}$ where $\rho$ is the solar wind density (fourth panel), is unreliable.

[36] The Swedish Institute of Space Physics, Lund, use ACE data to forecast the $K_{\mathrm{p}}$ index, and thus the problems with the measurement of the solar wind velocity suggest that their forecast is unreliable. In this example, the SPACECAST system continued forecasting without interruption by using the estimated $K_{\mathrm{p}}$ provided by the BGS. The estimated $K_{\mathrm{p}}$ is constructed from ground-based magnetometers and is thus more robust but provides a shorter warning period of up to approximately $2 \mathrm{~h}$. However, the fact that the SPACECAST system continued to operate automatically through this event illustrates the robustness of the system.

[37] Figure 4 shows the same event but for a higher energy of $>2 \mathrm{MeV}$. Again, there is substantial increase in the integral flux near $L^{*}=4$ and a gradual increase at lower $L^{*} \approx 3$ as the slot region starts to fill. However, in the second panel, there is a considerable difference between the model forecast at the location of GOES (red) and GOES measurements (pink). Other GOES data (not shown) revealed a large increase in the $>10 \mathrm{MeV}$ proton flux beginning around 03:00 UTC on 7 March, which exceeded the preexisting level by three orders of magnitude on 8 March 2012 and remained very high until well after the 11 March 2012. These data indicate that an SEP event took place as the same time as the geomagnetic storm. The guidance provided by the U.S. Space Weather Prediction Center states that GOES $>2$ $\mathrm{MeV}$ electron data can be unreliable during an SEP event as energetic protons can contaminate the electron measurements. The second panel in Figure 4 shows the ratio of the GOES $>2 \mathrm{MeV}$ flux to the $>10 \mathrm{MeV}$ proton 
HORNE ET AL.: RADIATION BELT FORECASTING

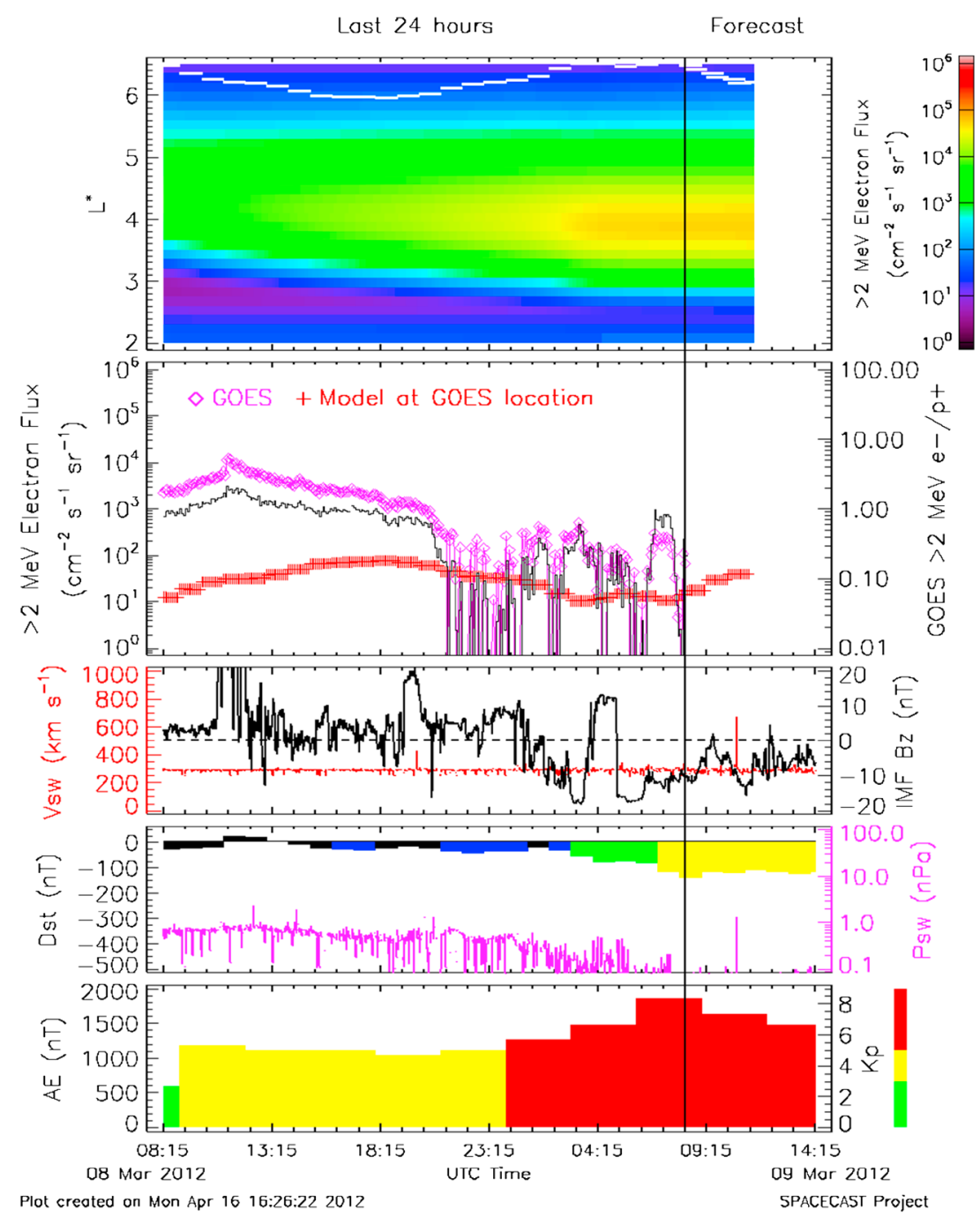

Figure 4. Same as Figure 3 except for $>2 \mathrm{MeV}$ integral electron flux. The second panel also includes the ratio of the GOES $>2 \mathrm{MeV}$ electron flux to the $>10 \mathrm{MeV}$ proton flux.

flux. This ratio is close to 1.0 and shows that the proton and electron flux varied in a very similar way which indeed suggests that the electron flux measurements are contaminated and unreliable. Note that by reference to Figure 3, the $>800 \mathrm{keV}$ electron flux does not show the same variability, suggesting that the $>800$ electron flux is relatively clean.

[38] Since the $>2 \mathrm{MeV}$ electron flux was contaminated with protons during this event, and much higher than it should be, any attempt to issue warnings using these data could lead to a false alarm. In contrast, the SPACECAST system of forecasts continued throughout the event, and the models which are not contaminated offer an opportunity to guard against false alarms. However, we caution that this study was for one event, many more studies are required to improve the forecasts for different types of events.

\section{Effects of Wave-Particle Interactions}

[39] The effects of wave-particle interactions on the radiation belts inside geostationary orbit have been demonstrated in several scientific studies [Shprits et al., 2006a, 2006b; Fok et al., 2008; Albert et al., 2009; Varotsou et al., 2005, 2008; Su et al., 2010]. However, the importance of wave-particle interactions outside geostationary orbit has not really been considered. To address this point, Figure 5 shows the results of the BAS model for the whole of March 2012 where the outer boundary has been extended to $L^{*}=8$ and the variation of the flux at the outer boundary with $K_{\mathrm{p}}$ is the same as before but where no wave-particle interactions are included between $L^{*}=6.5$ and 8 . In this case, the model is driven by the measured value of $K_{p}$, not the forecast of $K_{\mathrm{p}}$, and so it is not a forecasting run but instead provides a test of the model against data. The most striking 
HORNE ET AL.: RADIATION BELT FORECASTING

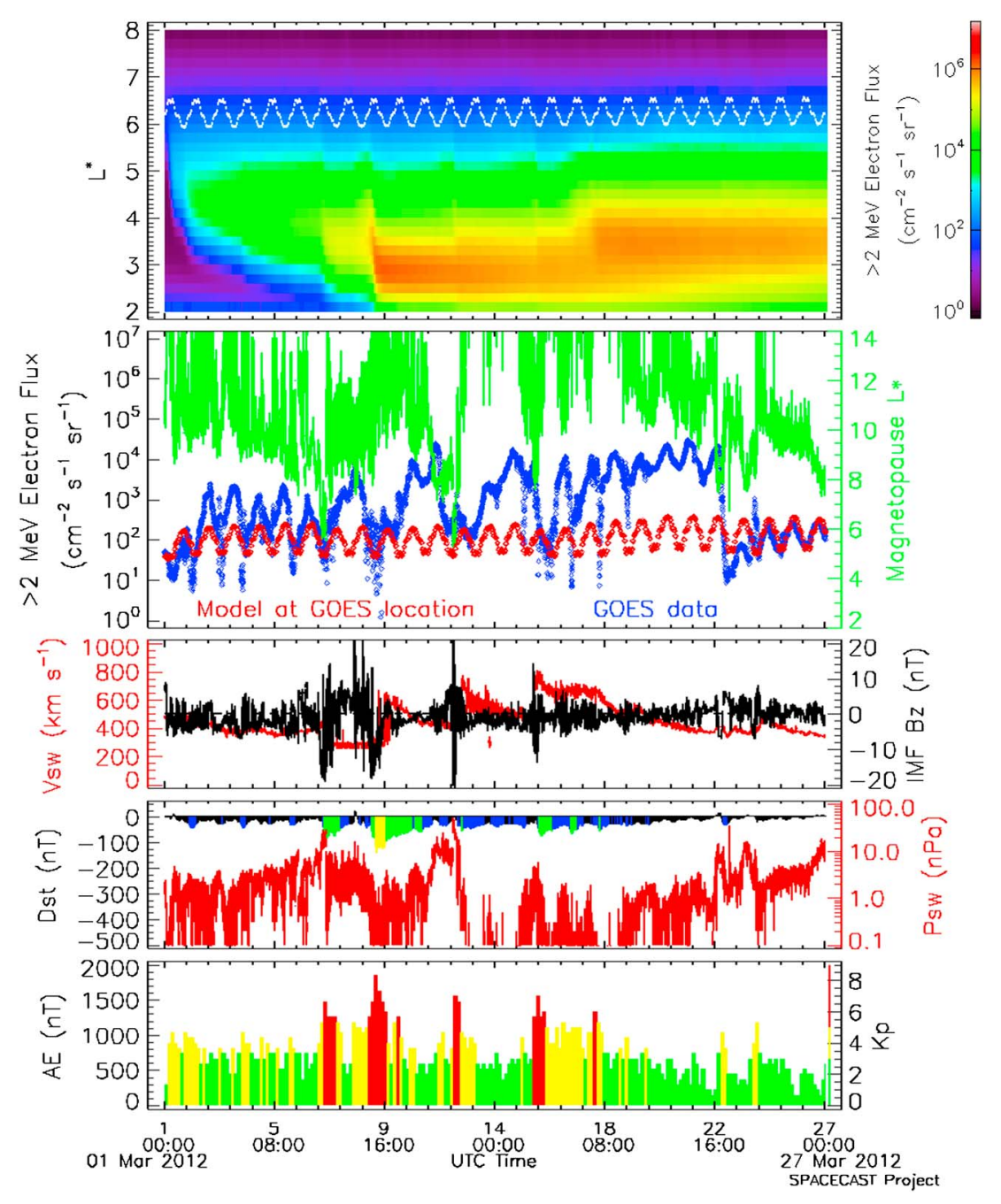

Figure 5. Results for the whole month of March, but without wave-particle interactions between $L^{*}=6.5-8$. The second panel also includes the location of the magnetopause calculated from the Shue model (in green).

feature is the increase in the electron flux on 8 and 9 March 2012 described previously (note the change in color scale). The slot region between the inner and outer radiation belts $L^{*}=2-3$ was filled during this event and took many days to decay toward the end of the month. The second panel shows that there is better agreement between the model and the GOES data during the initial part of the month, up until the 8th and 9th, when geomagnetic conditions were relatively quiet; and excepting the period from 7 to 12 March 2012 when the GOES data were contaminated by the SEP event, there is reasonable agreement between 12 and 14 March 2012. However, after 14 March, the model underestimates the electron flux by up to two orders of magnitude. After $14 \mathrm{March}$, the $D_{\text {st }}$ index indicates that there were some weak and moderate storms, for example, on 15 and 16 March, but there were no significant SEP events after 12 March. In fact, the GOES measured flux was up to two orders of magnitude higher than the forecast for a period of a few days after 10 and 14 March and was higher for a period of up to 5 days after 17 March. These periods of higher flux tend to follow periods of higher solar wind velocity, as shown in the third panel, but delayed by 1 or 2 days.

[40] Fast solar wind streams have been closely associated with increases in the relativistic electron flux [Paulikas and Blake, 1979; Baker et al., 1997; Iles et al., 2002; Miyoshi et al., 2004], but the mechanism by which they increase the radiation belt flux is unclear. Some studies have suggested that the fast solar wind flow along the outer boundary of the magnetopause excites ULF waves via Kelvin-Helmholz-type interaction and that these ULF waves propagate inside the magnetosphere and drive increased radial diffusion toward the Earth and hence electron acceleration. Of course, if the gradient in electron phase space density is inward, then this mechanism transports electrons away from the Earth and depletes the radiation belts. Other studies suggest that Alfvénic fluctuations in the fast solar wind drive substorms 
and convective electric fields that transport $\sim \mathrm{keV}$ energy electrons toward the Earth and excite whistler mode chorus waves. The chorus waves then accelerate a fraction of the electron population to enhance the radiation belts [Horne, 2007]. The models here provide a means of at least partially testing these ideas and improving predictions.

[41] In Figure 6, we have repeated the runs, but now wave-particle interactions due to chorus waves are also included between $L^{*}=6.5$ and 8 . The waves were included by simply extrapolating the wave properties to larger $L^{*}$. Radial diffusion is included in both the runs shown in Figures 5 and 6 . The most notable change is the increased variability in the electron flux at and beyond geostationary orbit. The second panel shows that there is a much better agreement with the GOES data after 10 and 14 March and that the model tends to track better the GOES data after 17 March but does not increase as quickly as the GOES measured flux. These results suggest that waveparticle interactions from geostationary orbit out to at least $L^{*}=8$ are a very important process and suggest that they can improve the forecasting substantially. At present, we have very little data on wave-particle interactions beyond geostationary orbit, but new wave models are being developed [e.g., Meredith et al., 2012].

\section{Solar Wind, Magnetopause, and Radiation Belt Coupling}

[42] It is interesting to note that there is a rapid drop in the GOES flux on the 22 March that is not captured in the modeling (Figures 5 and 6). Rapid flux dropouts like this are a subject of much debate. It has been suggested that these dropouts are due to losses by electromagnetic ion cyclotron (EMIC) waves [Thorne, 2010], although there does not appear to be much supporting evidence for the precipitating electron flux in low altitude satellite measurements [Horne et al., 2009; Meredith et al., 2011]. Alternatively, flux dropouts could be due to outward radial transport and losses at the magnetopause [Shprits et al., 2006a, 2006b].

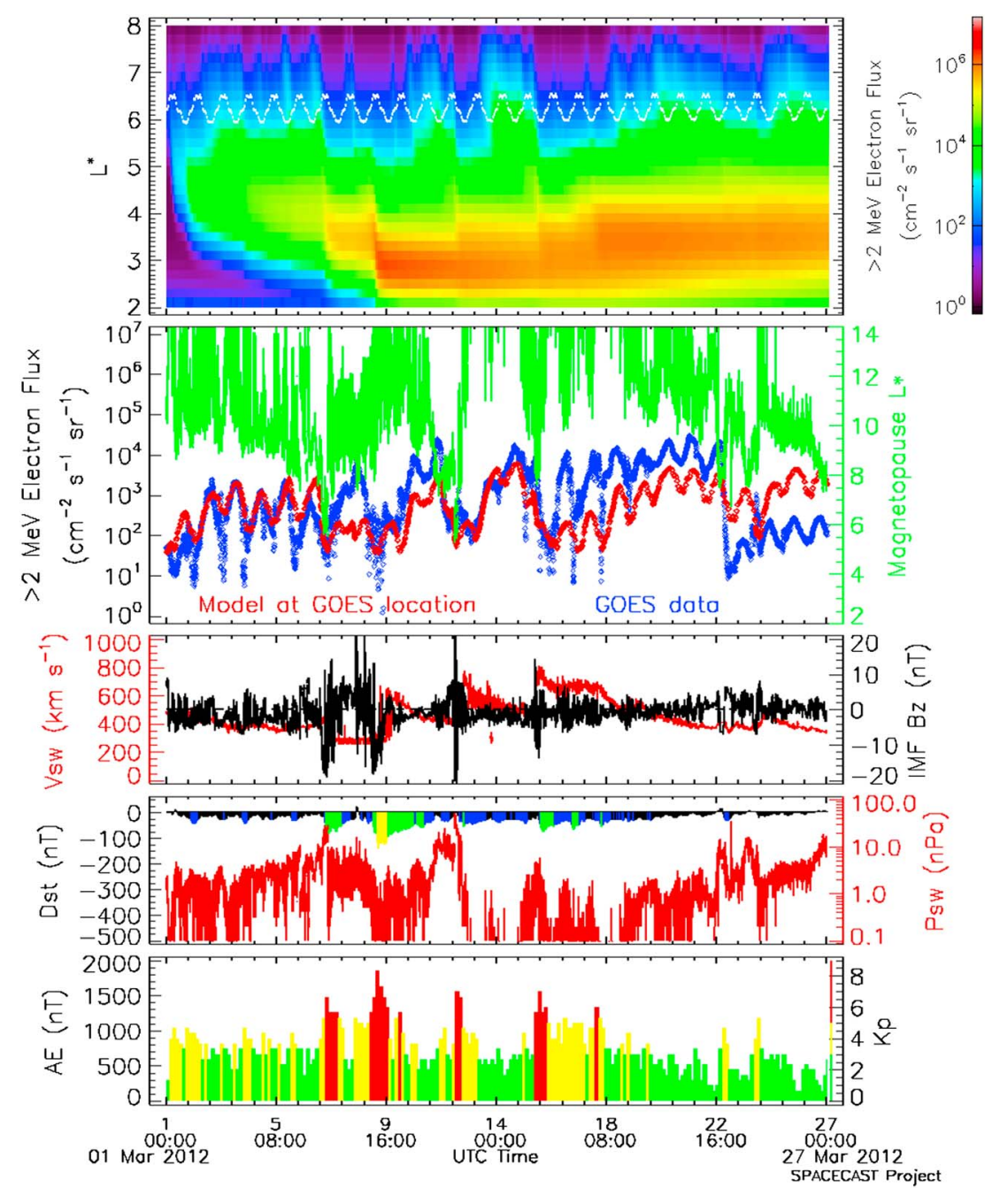

Figure 6. Same as Figure 5, but with wave-particle interactions between $L^{*}=6.5-8$. 
[43] Using the model of Shue et al. [1997, 1998] to calculate the location of the magnetopause, the green curve in the second panel shows that the rapid flux dropout on the 22 March occurred just after the solar wind pushed the magnetopause in to $L^{*}<8$. Similarly, the flux dropouts on the 11th and 15th also occurred after the magnetopause penetrated to $L^{*}<8$. This suggests that the magnetopause played an important role in the rapid electron flux dropout. We note that the magnetopause also reached $L^{*}$ $<8$ on 7 March and that there was a rapid drop in flux, but the flux did not remain low as in the other cases. It appears that the locations of the magnetopause, or other possible magnetic field reconfigurations, play an important but complicated role in radiation belt dynamics. Because EMIC wave are not included in our model, and because we do not yet include the effects of the magnetopause on radial diffusion and loss, we cannot determine which of these processes is most important. However, modeling suggests that including the effects of the magnetopause on radial transport and loss, or more generally, better coupling between the solar wind, and models of the magnetopause and the radiation belts, is likely to improve forecasting.

\section{Satellite Risk Index}

[44] Although the multipanel plots shown in Figures 3 and 4 are very instructive for scientific work, they are complicated and are not the most appropriate for use by satellite operators and service providers. The SWPC issues alerts when the $>2 \mathrm{MeV}$ electron flux at GOES exceeds $10^{3}$ particle flux units $\left(\mathrm{cm}^{-2} \mathrm{~s}^{-1} \mathrm{sr}^{-1}\right)$ for more than three consecutive 5 min periods. However, after discussion with satellite operators, we have chosen to provide a much simpler forecast in terms of a satellite risk index for internal charging. To set a general level of risk is very difficult since satellites have many differences in design, components, shielding, and operational procedures, and some may be more susceptible to the radiation environment than others. In the SPACECAST project, the risk is set according to past experience when an anomaly occurred repeatedly on a telecommunications satellite at geostationary orbit. The anomaly was an unwanted phantom command caused by internal ESD. A statistical analysis showed that the anomaly was caused by the accumulation of charge inside electronic components caused by high energy electrons $>2 \mathrm{MeV}$, and that it occurred when the electron fluence greater than $2 \mathrm{MeV}$ exceeded $10^{9} \mathrm{~cm}^{-2} \mathrm{sr}^{-1}$ during a 2-day period [Wrenn and Smith, 1996; Wrenn et al., 2002]. Internal charging requires time for the charge to accumulate to dangerous levels. Thus, setting the risk after 1 day rather than 2 days provides some level of advanced warning and time for operators monitor the environment for the next $24 \mathrm{~h}$. We have therefore set our risk index for geostationary orbit according to the electron fluence greater than $2 \mathrm{MeV}$ for a $24 \mathrm{~h}$ period for the following criteria

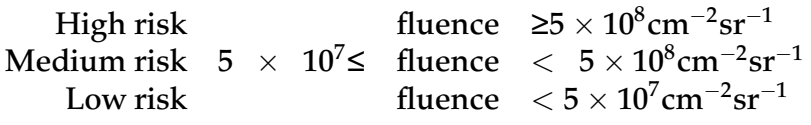

[45] The electron fluence is calculated from the previous $21 \mathrm{~h}$ and the forecast for the next $3 \mathrm{~h}$. The fluence and the risk index are updated every hour to provide high time resolution.

[46] The risk index is intended to provide a general guide for all satellites. However, if a satellite is particularly sensitive to anomalies at a different fluence level, then the risk index could be scaled up or down according to the fluence at which the anomalies occur. The daily electron fluence is also a convenient quantity since it is used in internal charging tools such as DICTAT [http://spaceenv.esa.int/index.php/dictat.html] to calculate internal charging of different types of material behind different thicknesses of aluminum shielding [Rodgers et al., 1998].

[47] As we have shown above, the radiation belt electron flux often drops rapidly at the beginning of a geomagnetic storm and then increases above the prestorm level for a period of 1-2 days. Not all storms increase the electron flux at geostationary orbit [Reeves et al., 2003], but instead the location of the peak radiation belt flux can move to lower $L^{*}$. For example, the peak electron flux moved to $L^{*}<3$ in the 2003 Halloween storm [Baker et al., 2004; Horne et al., 2005a], and simulations suggest the same behavior for an extreme space weather event [Shprits et al., 2011]. Since the risk of internal charging is closely related to the electron fluence, the SPACECAST system provides a risk index for internal spacecraft charging for three orbit types, GEO, MEO, and the slot region, to include the risk from extreme events.

[48] Figure 7 shows an example of the fluence for geostationary orbit for the 8 March 2012 event described earlier. The fluence obtained from the model, and the forecast of fluence (to the right of the vertical line), are both below the low-risk level and are thus colored green. The fluence obtained from the GOES satellite is an order of magnitude larger than that from the model, but during the period shown, the GOES data were contaminated by the SEP event and thus the data are unreliable. In contrast, Figure 8 shows that the fluence exceeded the high-risk level for MEO and the system forecast a high risk of internal charging (colored red). This forecast must be taken with some caution as most satellites in MEO have more shielding than those at GEO, and therefore the risk levels may be different. However, there is currently very little information to set the risk level for MEO, and so for now, we adopt the same risk levels. For lower MEO orbits, corresponding to the slot region, Figure 9 shows that the fluence was initially low as expected for this relatively benign region but started to increase after 09:15 UT, indicating that the slot region was starting to fill due to a mixture of inward electron transport and acceleration due to wave-particle interactions. This is more clearly evident at later times in Figures 5 and 6. Particle injections into the slot region are well known 


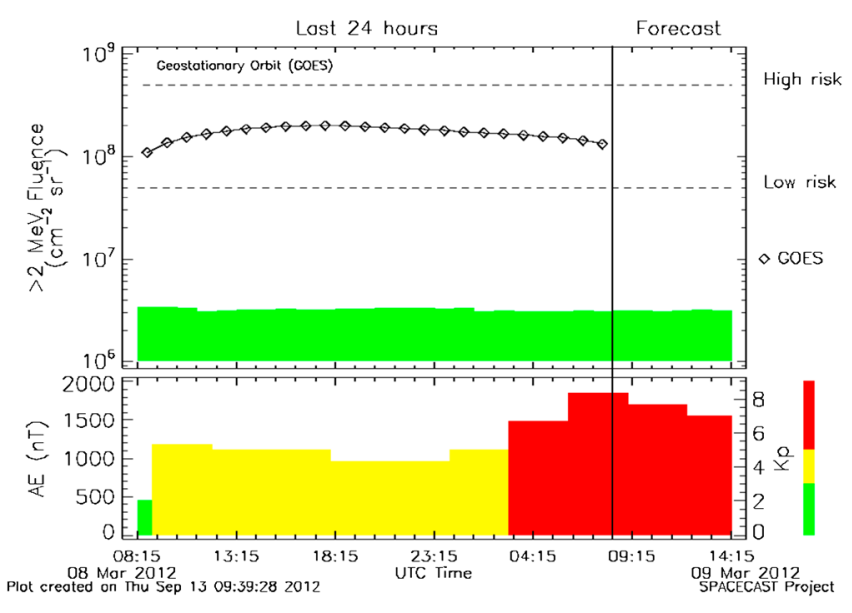

Figure 7. Top: the $24 \mathrm{~h}$ electron fluence $>2 \mathrm{MeV}$ for geostationary orbit. The fluence calculated from GOES 13 is shown by the diamonds and the model by the solid color fill. The $3 \mathrm{~h}$ forecast is to the right of the vertical line and the following $3 \mathrm{~h}$ forecast has been added. Bottom: the $K_{\mathrm{p}}$ index.

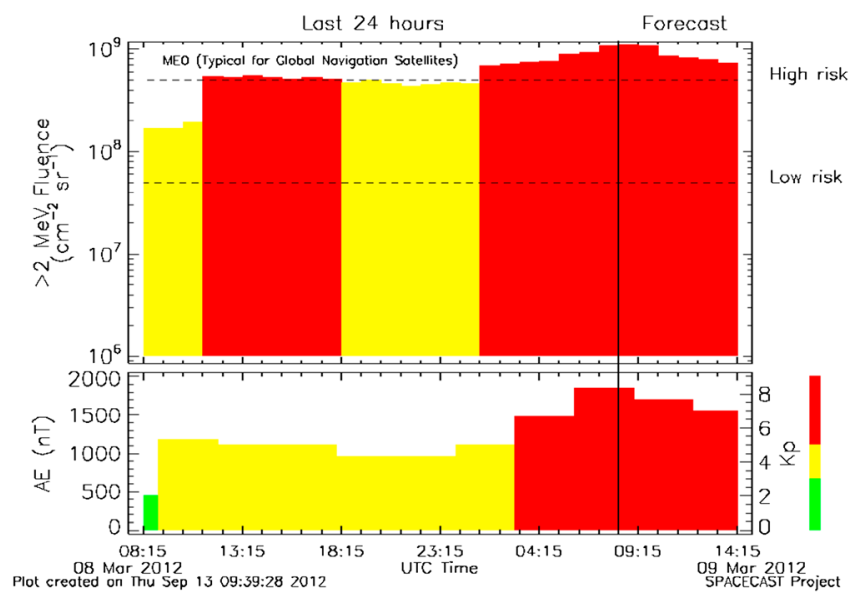

Figure 8. Top: the $24 \mathrm{~h}$ electron fluence $>2 \mathrm{MeV}$ for MEO. The $3 \mathrm{~h}$ forecast is to the right of the vertical line and the following $3 \mathrm{~h}$ forecast has been added. Bottom: the $K_{\mathrm{p}}$ index.

[Thorne et al., 2007] and are likely to be an important risk factor for new satellites designed to utilize this orbit.

\section{Timescale for Forecasting}

[49] Satellite operators would like to have a warning about space weather about a day or so ahead so that they can plan mitigating action. In general, reliable warnings on this timescale are not possible at present due to the need to measure the polarity of the interplanetary magnetic field, which can only be performed by spacecraft such as ACE in the solar wind. However, the forecasting timescale is more complicated than it first appears, and there can be

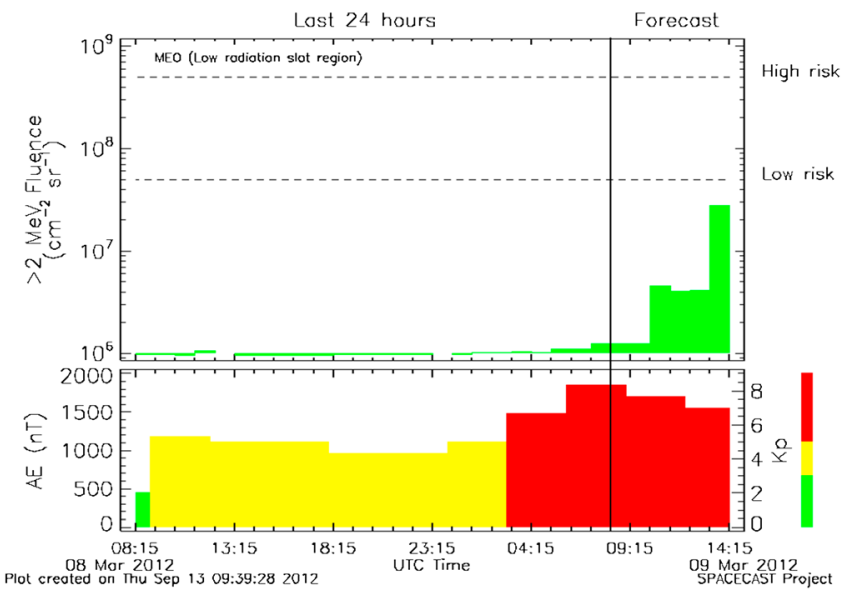

Figure 9. Top: the $24 \mathrm{~h}$ electron fluence $>2 \mathrm{MeV}$ for MEO in the slot region. The $3 \mathrm{~h}$ forecast is to the right of the vertical line and the following $3 \mathrm{~h}$ forecast has been added. Bottom: the $K_{\mathrm{p}}$ index.

conditions where a warning is possible on a timescale longer than $3 \mathrm{~h}$.

[50] For cases where solar wind speed and IMF Bz are fluctuating, and the IMF Bz does not have large negative excursions, it takes approximately $4 \mathrm{~h}$ for information about changes in the solar wind to be transferred to plasma sheet electrons, at energies of typically $1-30 \mathrm{keV}$, at geostationary orbit [Borovsky et al., 1998]. The observed timescale to accelerate electrons up to $\mathrm{MeV}$ energies is typically 1-2 days [Baker et al., 1994], which may take place via whistler mode chorus wave acceleration [e.g., Horne et al., 2005a, 2005b], inward radial diffusion driven by ULF waves [Elkington et al., 1999; Mann et al., 2004], or some other process such as acceleration by magnetosonic waves [Horne et al., 2007]. These time delays are in addition to the $\sim 60$ min time delay between the solar wind flowing from $\mathrm{L} 1$ to the magnetopause and help to make our $3 \mathrm{~h}$ forecasts more reliable than they might appear at first. The type of events where these conditions are more likely to apply are the fast solar wind streams emanating from coronal holes and which are associated with corotating interaction regions in the solar wind. These events drive long periods of turbulent solar wind where the IMF Bz fluctuates around zero rather than having large negative excursions [Tsurutani et al., 2006], and yet they are particularly effective in increasing the radiation belt electron flux [Horne et al., 2006]. It is thought that the fluctuating solar wind drives substorm activity lasting for days [Tsurutani and Gonzalez, 1987] and appears to be a key factor for increasing the electron radiation belts.

[51] On the other hand, for cases where the solar wind changes rapidly, on a timescale less than an hour or so, our forecasts are likely to be less reliable. These type of events are likely to include a fast CME, which pushes the magnetopause inside geostationary orbit or where the IMF $\mathrm{Bz}$ changes rapidly and acquires a large southward component that triggers a large geomagnetic storm. However, a 
fast CME compression and or a large storm usually lead to a rapid reduction in the $\mathrm{MeV}$ electron flux, or dropout, for a period of a few hours at the start of the storm. After the initial dropout, the flux tends to increase for a period of 1-2 days. At present, our models cannot deal with these types of events, but since high rather than low electron flux is more important for internal charging, this omission is probably not critical. It may be more important for surface charging.

[52] Our model cannot forecast changes associated with a very fast CME or interplanetary shock such as the one which occurred in March 1991 and which formed a new radiation belt within 2 min [Blake et al., 1992]. It is doubtful whether any system can do this at present. This type of event requires inclusion of other physical processes such as electron acceleration by an electric field pulse as a result of rapid compression of the geomagnetic field on a timescale less than the electron drift period [e.g., Li et al., 1993; Hudson et al., 1997].

\section{Discussion}

[53] The 7-9 March 2012, geomagnetic storms and SEP event are examples where space weather affected the very satellites that are used to predict and monitor space weather. These were not severe storms, but they provided an important test of the SPACECAST forecasting system. As the ACE and GOES satellite data became unreliable, the forecasting system was able to continue without interruption by using a nowcast of $K_{\mathrm{p}}$ from ground-based magnetometers. Several warnings were given by SWPC and other solar scientists about the CME and impending geomagnetic storm, and these were widely reported in the press. It is very important to guard against false alarms and develop a graduated system of warnings bearing in mind that we cannot predict the severity of the geomagnetic storm until we can measure the polarity of the interplanetary magnetic field. At present, this means measurement at the $\mathrm{L} 1$ position.

[54] During the events on 7-9 March, Sky Terra 1 reported problems including an outage of up to $24 \mathrm{~h}$ [http:// www.flightglobal.com/blogs/hyperbola/2012/03/spaceway3-temporarily-knocked.html]. Throughout the event, the SPACECAST system continued to forecast low risk of internal charging by $\mathrm{MeV}$ electrons at geostationary orbit, but other ground and satellite data provide evidence of significant substorm activity and plasma injections, which can cause surface charging. On 13 and 22 March, Spaceway 3 and GOES 15 also reported outages of a few hours and a few days, respectively. The actual cause of the anomalies has yet to be determined.

[55] The results from the two high energy radiation belt forecasting models sometimes pose a difficulty in that they can give different results. As each model is totally independent the differences may be due to the assumptions used for the physical processes in each model, the methods of solving the underlying equations, although the codes have been tested successfully for numerical stability, and differences in the way the boundary conditions are implemented. It is not always easy to show which model is the best. For example, one model may perform better for one set of geomagnetic conditions, whereas another may be better for other conditions. In the SPACECAST project, we have adopted a policy of only making one forecast, and thus we select the results considered to be the best for a particular set of conditions.

[56] We consider the use of two independent models a strength. Independent computer programs are commonly used in aircraft systems to ensure safety and reliability. We have adopted the same principle for our space weather forecasting. Only by comparing the results of each model against data for different conditions can we improve our understanding of the physics and evolve the models to make better forecasts. We expect the model results to converge as we improve them.

[57] The forecasting method presented here is based on physics-based models. It is also possible to forecast using other types of models such as empirical models [e.g., $\mathrm{Li}$ et al., 2001] and neural networks. These other types of model depend on the amount of data used to "train" them and can do a very good job of providing a reliable forecast where persistence is very strong. The advantage of physics-based models is that they are able to respond to the more unusual events and possibly more extreme events, provided the physical processes are understood and captured correctly. They can also reproduce the space environment with more confidence for orbits where there is little or no data. Meteorology shows that physics-based models now perform exceptionally well in terrestrial weather forecasting where the skill score is typically $80 \%$ of the perfect forecast [Lynch, 2008]. Thus, there is reason to believe that with more research, physics-based models can make more impact in space weather forecasting.

[58] There are several areas where our current radiation belt modeling can be improved. First, there are still very large uncertainties over wave-particle interactions and the conditions where they increase or decrease the radiation belt electron flux. We require more information on the different types of waves, such as chorus, plasmaspheric hiss, magnetosonic waves and EMIC waves, and for each wave type, more information on the frequency spectrum, the propagation direction, the latitude distribution, the magnetic local time and radial distribution of the waves, and how these properties change with geomagnetic activity. We have also used quasilinear theory to represent these waves in our model, but in reality these waves can be highly nonlinear and a theory that can take the microphysics of nonlinear wave-particle interactions and apply it on a global scale is required, a major challenge. Similar information is required on ULF waves, which drive electron transport across the geomagnetic field, particularly the magnetic local time dependence of the power spectra and the relative contributions of the toroidal and poloidal wavefields. A second major area of uncertainty is the coupling between the solar wind, magnetopause, and radiation belts that results in the rapid loss of electrons, shock-type acceleration 
events, and distorted geomagnetic fields. Models that can couple upstream to the solar wind would be better placed to give longer forecasting times. A third major area is the inclusion of lower-energy electrons and ions and their magnetic local time dependence. Including the injection of 1-100 keV electrons and ions via convective and inductive electric fields and via associated substorm events would provide a better source population for forecasting the higherenergy electrons, and the development of the ring current which would enable adiabatic and non adiabatic effects to be included self consistently. The list is not exhaustive.

\section{Summary and Conclusions}

[59] We present here the first European-led system to forecast the high energy electron radiation belts using physics-based models as part of the SPACECAST project. The system uses a forecast of the $K_{\mathrm{p}}$ index to drive two independent models, one in the UK and one in France, to forecast the high energy $(>300 \mathrm{keV})$ electron flux throughout the outer radiation belt for a period of up to $3 \mathrm{~h}$ ahead. The system has three unique features. First, it uses physics-based models, which include wave-particle interactions to provide the forecasts. This is the first time wave-particle interactions and radial diffusion have been combined to produce preoperational forecasts. Second, it provides a forecast for the whole of the outer radiation belt, including geostationary orbit where most commercial satellites operate, MEO where most GNSS operate, such as GPS (USA), Galileo (Europe), GLONASS (Russia), and Compass (China), and the slot region between the inner and the outer radiation belts where the radiation environment is usually benign, but can increase rapidly during storms, and where the number of satellites is expected to grow. Third, the SPACECAST system is a truly international effort and uses data from U.S. satellites, geomagnetic indices from Europe and Japan, and a network of European forecasting and computer models.

[60] To help satellite operators interpret the results, the forecasts are presented in the form of a risk index for internal satellite charging based on the 24 h electron fluence $>2$ $\mathrm{MeV}$. The forecasts are updated every hour, and the system has run automatically since November 2011 with only one service interruption as of August 2012. An important test of the system was the 7-9 March 2012 geomagnetic storm, where solar wind velocity data from the ACE spacecraft became unreliable due to an SEP event and the $>2$ $\mathrm{MeV}$ electron flux measured by GOES was contaminated with protons. The forecasting system continued without interruption by using a nowcast of the $K_{\mathrm{p}}$ index derived from ground-based magnetometers provided by the BGS. The forecasting system was able to forecast the electron flux to within a factor of 2 during the initial part of the storm, and to within a factor of 10 during the later part of the storm. However, we caution that this was for one event, many more studies are required to improve the forecasts for different types of events. This is the subject of future work.
[61] Modeling shows that including wave-particle interactions in the outer part of the radiation belt, between $L^{*}=6.5$ and 8 , results in a much better agreement between the models and the $>800 \mathrm{keV}$ and $>2 \mathrm{MeV}$ electron flux at geostationary orbit. Thus, including wave-particle interactions beyond geostationary orbit is likely to improve forecasting significantly.

[62] Modeling also shows that an inward motion of the magnetospause to lower $L^{*}$ is closely associated with rapid dropouts in the relativistic electron flux at geostationary orbit. The dropouts are observed although the magnetopause may not actually reach geostationary orbit but comes to within 1-2 Re of it. It is not yet possible to determine whether this is due to outward transport and loss at the magnetopause [Shprits et al., 2006a, 2006b] or whether the magnetopause excites other types of wave-particle interactions such as EMIC waves that cause the loss [e.g., Thorne, 2010]. However, better coupling between the solar wind and the models of the magnetopause and the radiation belts is likely to improve forecasting significantly.

[63] Space weather forecasting using physics-based models is at a very early stage. To develop better quantitative forecasting and even nowcasting, much more work is required to develop better initial conditions and boundary conditions, to develop better models of wave-particle interactions which better capture the wave characteristics, plasma density models which affect loss and acceleration, better models of ULF waves which drive high energy electron transport, electric field models that transport the low energy seed electrons, and better magnetic fields models, which are coupled to the ring current and the solar wind. This is a formidable challenge, but with new satellites such as the NASA Van Allen probes mission, there is every prospect of making substantial progress with physicsbased models over the next few years, following the example of terrestrial weather forecasting.

\section{Appendix A}

[64] The two radiation belt models calculate the evolution of the electron phase space density in the radiation belts from a diffusion equation given by Schulz and Lanzerotti [1974]. The equation can be written as

$$
\begin{aligned}
\frac{\partial f}{\partial t}= & \frac{1}{G} \frac{\partial}{\partial \alpha_{\mathrm{eq}}}\left[G\left(D_{\alpha_{\mathrm{eq}} \alpha_{\mathrm{eq}}} \frac{\partial f}{\partial \alpha_{\mathrm{eq}}}+D_{\alpha_{\mathrm{eq}} E} \frac{\partial f}{\partial E}\right)\right] \\
& +\frac{1}{G} \frac{\partial}{\partial E}\left[G\left(D_{\mathrm{EE}} \frac{\partial f}{\partial E}+D_{E \alpha_{\mathrm{eq}}} \frac{\partial f}{\partial \alpha_{\mathrm{eq}}}\right)\right] \\
& +L^{2} \frac{\partial}{\partial L}\left[\frac{1}{L^{2}} D_{\mathrm{LL}} \frac{\partial f}{\partial L}\right]-\left(\frac{f}{0.5 \tau_{b}}\right)_{\text {losscone }}
\end{aligned}
$$

where

$$
G=T\left(\alpha_{\mathrm{eq}}\right) \sin 2 \alpha_{\mathrm{eq}}\left(E+E_{0}\right) \sqrt{E\left(E+2 E_{0}\right)}
$$

$f\left(J_{1}, J_{2}, J_{3}, t\right)$ is the bounce and drift averaged distribution function, $E$ and $E_{0}$ are the electron energy and rest mass 
energy, $\alpha_{\text {eq }}$ is the equatorial pitch angle, $L$ is McIlwain's $L$ shell parameter $\left(R=\mathrm{LR}_{\mathrm{e}} \cos ^{2} \lambda_{\mathrm{m}}\right.$, where $\lambda_{m}$ is the magnetic latitude and $R_{\mathrm{e}}$ is the Earth's radius $=6370 \mathrm{~km}$ ) and $\tau_{b}$ is the electron bounce period. The derivatives with respect to $L$ are for fixed $J_{1}$ and $J_{2}$, whereas the derivatives with respect to $\alpha_{\mathrm{eq}}$ and $E$ are for fixed $L$. For a dipole field, a very good approximation to $T\left(\alpha_{\mathrm{eq}}\right)$ is [Schulz and Lanzerotti, p19, 1974]

$$
T\left(\alpha_{\mathrm{eq}}\right)=1.3802-0.3198\left(\sin \alpha_{\mathrm{eq}}+\sin ^{\frac{1}{2}} \alpha_{\mathrm{eq}}\right)
$$

[65] The first four terms on the right-hand side of equation A1 represent diffusion in pitch angle and energy due to wave-particle interactions, the fifth term represents transport across the magnetic field as a result of radial diffusion driven by ULF waves, and the last term represents losses into the atmosphere for particles that are diffused into the loss cone. The timescales for loss is half the electron bounce period and is energy dependent. The diffusion coefficients are for pitch angle $D_{\alpha_{\mathrm{eq}} \alpha_{\mathrm{eq}}}$ energy $D_{\mathrm{EE}}$ mixed pitch angle-energy $D_{\alpha_{\mathrm{eq}} E}$, and radial diffusion $D_{\mathrm{LL}}$ and are scaled according to $K_{\mathrm{p}}$. At present, the Salambô model includes the cross terms but the BAS model does not.

[66] More details of the models are given by Varotsou et al. [2005, 2008].

[67] Acknowledgments. The SPACECAST forecasting system uses data from several sources to produce the radiation belt forecasts. In particular, the authors thank the ACE SWEPAM and MAG instrument teams and the ACE Science Center for providing plasma and magnetic field data on the solar wind; the National Oceanic and Atmosphere Administration for real-time data from the GOES and POES satellites; the Swedish Institute of Space Physics, Lund, for providing forecasts of the $K_{\mathrm{p}}$ and $D_{\mathrm{st}}$ indices; the BGS for providing an estimated $K_{\mathrm{p}}$ magnetic index in near real time; the Regional Warning Centre, Kyoto, for the preliminary and quick look $D_{\text {st }}$ index; the Helmholdz Centre, Potsdam, for providing an archive of the $K_{\mathrm{p}}$ index; the National Geophysical Data Centre for providing an archive of the $D_{\mathrm{st}}$ index; and the European Space Agency for use of their open data interface. The research leading to these results has received funding from the European Union Seventh Framework Programme (FP7/2007-2013) under grant agreement no. 262468. They also acknowledge financial support from the UK Natural Environment Research Council and the French Aerospace Research Laboratory (ONERA).

\section{References}

Abel, B., and R. M. Thorne (1998), Electron scattering loss in Earth's inner magnetosphere 1. Dominant physical processes, J. Geophys. Res., 103, 2385-2396.

Albert, J. M., N. P. Meredith, and R. B. Horne (2009), Threedimensional diffusion simulation of outer radiation belt electrons during the October 9, 1990, magnetic storm, J. Geophys. Res., 114, A09214, doi:10.1029/2009JA014336.

Allen, J. (2012), The Galaxy 15 Anomaly: Another Satellite in the Wrong Place at a Critical Time, Space Weather, 8, S06008, doi:10.1029/2010SW000588.

Baker, D. N., J. B. Blake, L. B. Callis, J. R. Cummings, D. Hovestadt, S. G. Kanekal, B. Klecker, R. A. Mewaldt, and R. D. Zwickl (1994), Relativistic electron acceleration and decay times in the inner and outer radiation belts: SAMPEX, Geophys. Res. Lett., 21, 409-412.

Baker, D. N., et al. (1997), Recurrent geomagnetic storms and relativistic electron enhancements in the outer magnetosphere: ISTP coordinated measurements, J. Geophys. Res., 102, 14,141-14,148.
Baker, D. N., J. H. Allen, S. G. Kanekal, and G. D. Reeves (1998), Disturbed space environment may have been related to pager satellite failure, EOS transactions, AGU, 79, 477.

Baker, D. (2001), Satellite anomalies due to space storms, in Space Storms and Space Weather Hazards, edited by I. A. Daglis, ch. 10, pp. 251-284, Kluwer, Dordrecht, The Netherlands,

Baker, D. N., S. G. Kanekal, X. Li, S. P. Monk, J. Goldstein, and J. L. Burch (2004), An extreme distortion of the Van Allen belt arising from the 'Hallowe'en' solar storm in 2003, Nature, 432, 878-881.

Beutier, T., and D. Boscher (1995), A three-dimensional analysis of the electron radiation belt by the Salammbô code, J. Geophys. Res., 100, $14,853$.

Blake, J. B., W. A. Kolasinski, R. W. Fillius, and E. G. Mullen (1992), Injection of electrons and protons with energies of tens of $\mathrm{MeV}$ into $L<3$ on 24 March 1991, Geophys. Res. Lett., 19, 821-824.

Bodeau, M. (2007), Killer electrons from the angry Sun did not stop the pagers, Space Weather, 5, S03006, doi:10.1029/2006SW000266.

Bodeau, M. (2010) High energy electron climatology that supports deep charging risk assessment in GEO, in 48th AIAA Aerospace Sciences Meeting.

Borovsky, J. E., M. F. Thomsen, and R. C. Elphic (1998), The driving of the plasma sheet by the solar wind, J. Geophys. Res., 103, $17,617-17,63$.

Brautigam, D. H. and J. M. Albert (2000), Radial diffusion analysis of outer radiation belt electrons during the October 9, 1990, magnetic storm, J. Geophys. Res., 105, 291-309.

Brekke, P., (2004), Space weather effects on SOHO and its space weather warning capabilities, in Effects of Space Weather on Technology Infrastructure, edited by I. A. Daglis, ch. 6, pp. 109-122, Kluwer, Dordrecht, The Netherlands.

Cabinet Office, (2012), National risk register of civil emergencies, 22 Whitehall, London SW1A 2WH, www.cabinetoffice.gov.uk

Cannon, P., et al. (2013), Extreme space weather: impacts on engineered systems and infrastructure, Royal Academy of Engineering, Carlton House Terrace, London.

Cliver, E. W., J. Feynman and H. B. Garrett (1990), An Estimate of the Maximum Speed of the Solar Wind, 1938-1989, J. Geophys. Res., 95, A10, 17,103-17,112.

Denig, W., et al. (2011), Space Weather conditions at the time of the Galaxy 15 Anomaly, 2011 Space Weather Workshop, 26-29 April 2011.

Elkington, S. R., M. K. Hudson, and A. A. Chan (1999), Acceleration of relativistic electrons via drift resonant interactions with toroidalmode Pc-5 ULF oscillations, Geophys. Res. Lett., 26, 3273-3276.

Fennell, J. F., H. C. Koons, J. L. Roeder, and J. B. Blake (2001), Spacecraft Charging: Observations and Relationship to Satellite Anomalies, in Proceedings of 7 th Spacecraft Charging Technology Conference, 476, pp. 279-285, European Space Agency, Noordwijk, Netherlands.

Fennell, J. F., et al. (2010), Charge deposition behind known shielding in a highly inclined orbit, 11th Spacecraft Charging Technology Conference, Albuquerque, NM, Sept 2010.

Ferguson, D. C., W. F. Denig, and J. V. Rodriguez (2011), Plasma conditions during the Galaxy 15 anomaly and the possibility of ESD from subsurface charging, Proceedings of the 49th AIAA Aerospace Sciences meeting in Orlando, Florida, 4-7 Jan 2011.

Fok, M.-C., R. B. Horne, N. P. Meredith, and S. A. Glauert (2008), Radiation Belt Environment model: Application to space weather nowcasting, J. Geophys. Res., 113, A03S08, doi:10.1029/2007JA012558.

Friedel, R. H. W., S. Bourdarie, and T. E. Cayton (2005), Intercalibration of magnetospheric energetic electron data, Space Weather, 3, S09B04, doi:10.1029/2005SW000153.

Frederickson, A. R. (1996), Upsets related to spacecraft charging, IEEE Trans. Nucl. Sci., 43(2), 426-441.

Green, J. C., and M. G. Kivelson (2004), Relativistic electrons in the outer radiation belt: Differentiating between acceleration mechanisms, J. Geophys. Res., 109, A03213, doi:10.1029/2003JA010153.

Gubby, R., and J. Evans (2002), Space Environment effects and satellite design, J. Atmos. Solar Terr. Phys., 64, 1723-1733.

Hastings, D., and H. Garrett (1996), Spacecraft-Environment Interactions, Cambridge University Press, Cambridge, UK.

Horne, R. B., and R. M. Thorne (1998), Potential waves for relativistic electron scattering and stochastic acceleration during magnetic storms, Geophys. Res. Lett., 25, 3011-3014.

Horne, R. B., et al. (2005a), Wave acceleration of electrons in the Van Allen radiation belts, Nature, 437, 227-230, doi:10.1038/nature03939. 
Horne, R. B., R. M. Thorne, S. A. Glauert, J. M. Albert, N. P. Meredith, and R. R. Anderson (2005b), Timescale for radiation belt electron acceleration by whistler mode chorus waves, J. Geophys. Res., 110, A03225, doi:10.1029/2004JA010811.

Horne, R. B., N. P. Meredith, S. A. Glauert, A. Varotsou, R. M. Thorne, Y. Y. Shprits, and R. R. Anderson (2006), Mechanisms for the acceleration of radiation belt electrons, in Recurrent Magnetic Storms: Corotating Solar Wind Streams, Geophys. Monogr. Ser., vol. 167, edited by B. T. Tsurutani, R. L. McPherron, W. D. Gonzalez, G. Lu, J. H. A. Sobral, and N. Gopalswamy, pp. 151-173, AGU, Washington, D.C.

Horne, R. B. (2007), Plasma astrophysics: Acceleration of killer electrons, Nat. Phys., 3, 590-591.

Horne, R. B., R. M. Thorne, S. A. Glauert, N. P. Meredith, D. Pokhotelov and O. Santolik (2007), Electron acceleration in the Van Allen radiation belts by fast magnetosonic waves, Geophys. Res. Lett., 34, L17107, doi:10.1029/2007GL030267.

Horne, R. B., M. M. Lam, and J. C. Green (2009), Energetic electron precipitation from the outer radiation belt during geomagnetic storms, Geophys. Res. Lett., 36, L19104, doi:10.1029/2009GL040236.

Hudson, M. K., S. R. Elkington, J. G. Lyon, V. A. Machenko, I. Roth, M. Temerin, J. B. Blake, M. S. Gussenhoven, and J. R. Wygant (1997), Simulation of radiation belt formation during sudden storm commencements, J. Geophys. Res., 102, 14,087.

Iles, R. H. A., A. N. Fazakerley, A. D. Johnstone, N. P. Meredith, and P. Bühler (2002), The relativistic electron response in the outer radiation belt during magnetic storms, Ann. Geophys., 20, 957-965.

Iucci, N., et al. (2005), Space weather conditions and spacecraft anomalies in different orbits, Space Weather, 3, S01001, doi:10.1029/ 2003SW000056.

Koons, H. C., and J. F. Fennell (2006), Space weather effects on communications satellites, The Radio Science Bulletin, International Union of Radio Science (URSI), 316, March 27-41.

Kunstadter, C. (2012), View from the Leading Edge, presentation given at the World Space Risk Forum, 2012, Dubai, http://worldspaceriskforum.com/2012/presentations/

Lanzerotti, L. J., C. Breglia, D. W. Maurer, G. K. Johnson, III, and C. G. Maclennan (1998a), Studies of spacecraft charging on a geosynchronous telecommunications satellite, Ad Space Res., 22, 79-82.

Lanzerotti, L. J., K. LaFleur, C. G. Maclennan, and D. W. Maurer (1998b), Geosynchronous spacecraft charging in January 1997, Geophys. Res. Lett., 25(15), 2967-2970, doi:10.1029/98GL00987.

Li, X., I. Roth, M. Temerin, J. Wygant, M. K. Hudson, and J. B. Blake (1993), Simulation of the prompt energization, and transport of radiation belt particles during the March 231991 SSC, Geophys. Res. Lett., 20, 2423.

Li, X., M. Temerin, D. N. Baker, G. D. Reeves, and D. Larson (2001), Quantitative prediction of radiation belt electrons at geostationary orbit based on solar wind measurements, Geophys. Res. Lett., 28, 1887-1890.

Loewe, C. A., and G. W. Prölss (1997), Classification and mean behavior of magnetic storms, J. Geophys. Res., 102 14,209-14,213.

Lynch, P. (2008), The origins of computer weather prediction and climate modeling, J. Comput. Phys., 227, 3431-3444.

Lyons, L. R., and R. M. Thorne (1973), Equilibrium structure of radiation belt electrons, J. Geophys. Res., 78, 2142-2149.

Mann, I. R., T. P. O'Brien, and D. Milling (2004), Correlations between ULF wave power, solar wind speed, and relativistic electron flux in the magnetosphere: solar cycle dependence, J. Atmos. Solar Terr. Phys., 66, 187-198.

Meredith, N. P., M. Cain, R. B. Horne, R. M. Thorne, D. Summers, and R. R. Anderson, (2003), Evidence for chorus driven electron acceleration to relativistic energies from a survey of geomagnetically disturbed periods, J. Geophys. Res., 108(A6), 1248, doi:10.1029/2002JA009764.

Meredith, N. P., R. B. Horne, S. A. Glauert, D. N. Baker, S. G. Kanekal, and J. M. Albert (2009), Relativistic electron loss timescales in the slot region, J. Geophys. Res., 114, A03222, doi:10.1029/2008JA013889.

Meredith, N. P., R. B. Horne, M. M. Lam, M. H. Denton, J. E. Borovsky, and J. C. Green (2011), Energetic electron precipitation during highspeed solar wind stream driven storms, J. Geophys. Res., 116, A05223, doi:10.1029/2010JA016293.

Meredith, N. P., R. B. Horne, A. Sicard-Piet, D. Boscher, K. H. Yearby, W. Li, and R. M. Thorne (2012), Global model of lower band and upper band chorus from multiple satellite observations, J. Geophys. Res., 117, A10225, doi:10.1029/2012JA017978.
Miyoshi, Y. S., V. K. Jordanova, A. Morioka, and D. S. Evans (2004), Solar cycle variations of the electron radiation belts: Observations and radial diffusion simulation, Space Weather, 2, S10S02, doi:10.1029/2004SW000070.

National Academy of Sciences, (2008), Severe space weather events, Understanding societal and economic impacts.

O'Brien, T. P. (2009), SEAES-GEO: A spacecraft environmental anomalies expert system for geosynchronous orbit, Space Weather, 7, S09003, doi:10.1029/2009SW000473.

Odenwald, S. F., and J. L. Green (2007), Forecasting the impact of an 1859-caliber superstorm on geosynchronous Earth-orbiting satellites: Transponder resources, Space Weather, 5, S06002, doi:10.1029/ 2006SW000262.

Paulikas, G. A., and J. B. Blake (1979), Effects of the solar wind on magnetospheric dynmaics: Energetic electrons at the synchronous orbit, in Quantitative modelling of Magnetospheric processes, Geophys. Mongr. Ser., vol. 21, edited by W. P. Olsen, pp. 180-202, AGU, Washington D.C.

Reeves, G. D., K. L. McAdams, R. H. W. Friedel, and T. P. O’Brien (2003), Acceleration and loss of relativistic electrons during geomagnetic storms, Geophys. Res. Lett., 30, doi:10.1029/2002GL016513.

Rodgers D. J., K. A. Ryden, G. L. Wrenn, P. M. Latham, J. Sorensen, and L. Levy (1998), An Engineering Tool for the Prediction of Internal Dielectric Charging, 6th Spacecraft Charging Technology Conference, Hanscom.

Satellite Industry Association, (2012), State of the Satellite Industry Report 2012, www.sia.org/state-of-the-satellite-industry-report/

Schulz, M., and L. Lanzerotti (1974), Particle diffusion in the radiation belts, Springer, New York.

Shprits, Y. Y., R. M. Thorne, R. B. Horne, S. A. Glauert, M. Cartwright, C. T. Russell, D. N. Baker, and S. G. Kanekal (2006a), Acceleration mechanism responsible for the formation of the new radiation belt during the 2003 Halloween Solar storm, Geophys. Res. Lett., 33, L05104, doi:10.1029/2005GL024256.

Shprits, Y. Y., R. M. Thorne, R. Friedel, G. D. Reeves, J. Fennell, D. N. Baker, and S. G. Kanekal (2006b), Outward radial diffusion driven by losses at magnetopause, J. Geophys. Res., 111, A11214, doi:10.1029/2006JA011657.

Shprits, Y. Y., D. Subbotin, and B. Ni (2009), Evolution of electron fluxes in the outer radiation belt computed with the VERB code, $J$. Geophys. Res., 114, A11209, doi:10.1029/2008JA013784.

Shprits, Y. Y., D. Subbotin, B. Ni, R. Horne, D. Baker, and P. Cruce (2011), Profound change of the near-Earth radiation environment caused by solar superstorms, Space Weather, 9, S08007, doi:10.1029/ 2011SW000662.

Shue, J. H., J. K. Chaoa, H. C. Fu, C. T. Russell, P. Song, K. K. Khurana, and H. J. Singer (1997), A new functional form to study the solar wind control of the magnetopause size and shape, J. Geophys. Res., 102, A5, 9497-9511.

Shue, J. H., et al. (1998), Magnetopause location under extreme solar wind conditions, J. Geophys. Res., 103, A8, 17,691-17,700.

$\mathrm{Su}, \mathrm{Z}$., F. Xiao, H. Zheng, and S. Wang (2010), STEERB: A threedimensional code for storm-time evolution of electron radiation belt, J. Geophys. Res., 115, A09208, doi:10.1029/2009JA015210.

Summers, D., R. M. Thorne, and F. Xiao (1998), Relativistic theory of wave-particle resonant diffusion with application to electron acceleration in the magnetosphere, J. Geophys. Res., 103, 20,487.

The Economist (2012), High Frequency Trading, The Fast and the Furious, 25 Feb, http://www.economist.com/node/21547988

The Futron Corporation (2002), Satellite Insurance Rates on the RiseMarket Correction or Overreaction? July 10.

Thorne, R. M., Y. Y. Shprits, N. P. Meredith, R. B. Horne, W. Li, S. Liu, L. R. Lyons, and R. R. Anderson (2007), Refilling of the slot region between the inner and outer electron radiation belts during geomagnetic storms, J. Geophys. Res., 112, A06203, doi:10.1029/2006JA012176.

Thorne, R. M. (2010), Radiation belt dynamics: The importance of wave-particle interactions, Geophys. Res. Lett., 37, L22107, doi:10.1029/2010GL044990.

Tsurutani, B. T., and W. D. Gonzalez (1987), The cause of highintensity long-duration continuous AE activity (HILDCAAS): Interplanetary Alfven wave trains, Planet. Space Sci., 35, 405.

Tsurutani, B. T., W. D. Gonzalez, G. S. Lakhina, and S. Alex (2003), The extreme magnetic storm of 1-2 September 1859, J. Geophys. Res., 108(A7), 1268, doi:10.1029/2002JA009504. 


\section{HORNE ET AL.: RADIATION BELT FORECASTING}

Tsurutani, B. T., N. Gopalswamy, R. L. McPherron, W. D. Gonzalez, G. Lu, and F. L. Guarnieri (2006), Magnetic storms caused by corotating solar wind streams, in Recurrent Magnetic Storms: Corotating Solar Wind Streams, Geophys. Monogr. Ser., vol. 167, edited by B. T. Tsurutani, R. L. McPherron, W. D. Gonzalez, G. Lu, J. H. A. Sobral, and N. Gopalswamy, pp. 1-17, AGU, Washington, D. C.

Varotsou, A., D. Boscher, S. Bourdarie, R. B. Horne, S. A. Glauert, and N. P. Meredith (2005), Simulation of the outer radiation belt electrons near geosynchronous orbit including both radial diffusion and resonant interaction with whistler-mode chorus waves, Geophys. Res. Lett., 32, L19106, doi:10.1029/ 2005GL023282.

Varotsou, A., D. Boscher, S. Bourdarie, R. B. Horne, N. P. Meredith, S. A. Glauert, and R. H. Friedel (2008), Three-dimensional test simulations of the outer radiation belt electron dynamics including electron-chorus resonant interactions, J. Geophys. Res., 113, A12212, doi:10.1029/2007JA012862.

Webb, D. F., and J. H. Allen (2004), Spacecraft and ground anomalies related to the October-November 2003 solar activity, Space Weath., 2, doi:10.1029/2004SW000075.

Wrenn, G. L. (1995), Conclusive Evidence for Internal Dielectric Charging Anomalies on Geosynchronous Communications Spacecraft, J. Spacecraft and Rockets, 32, 514-520.

Wrenn G. L., and R. J. K. Smith (1996), The ESD threat to GEO satellites: Empirical models for observed effects due to both surface and internal charging, ESA symposium proceedings on 'Environment Modelling for space-based Applications', ESTEC, Noorwijk, NL, SP-392, p.121.

Wrenn, G. L., D. J. Rodgers, and K. A. Ryden (2002), A solar cycle of spacecraft anomalies due to internal charging, Ann Geophys., 20, 953-956. 